\title{
The gut microbiome of 7-37 month old children from The Gambia shows the development of a distinct non-industrial Prevotella-based trophic network
}

\author{
Marcus de Goffau \\ Wellcome Sanger Institute
}

Amadou Jallow

Medical Research Council Unit The Gambia at the London School of Hygiene \& Tropical Medicine, Banjul, The Gambia.

\section{Chilel Sanyang}

Medical Research Council Unit The Gambia at the London School of Hygiene \& Tropical Medicine, Banjul, The Gambia

\section{Andrew Prentice}

MRC Unit The Gambia at London School of Hygiene \& Tropical Medicine https://orcid.org/0000-00015389-451X

\section{Julian Parkhill}

University of Cambridge https://orcid.org/0000-0002-7069-5958

\section{Dora Pereira}

Department of Pathology, University of Cambridge, Tennis Court Road, Cambridge CB2 1QP, U.K.

\section{Josef Wagner ( $\sim$ Josef.Wagner@mh.org.au )}

Wellcome Sanger Institute https://orcid.org/0000-0003-1204-7765

\section{Article}

Keywords: African children, Prevotella, microbiome development, trophic network

Posted Date: March 2nd, 2021

DOl: https://doi.org/10.21203/rs.3.rs-220931/v1

License: (c) (1) This work is licensed under a Creative Commons Attribution 4.0 International License. Read Full License 
Version of Record: A version of this preprint was published at Nature Microbiology on December 31st, 2021. See the published version at https://doi.org/10.1038/s41564-021-01023-6. 
1 The gut microbiome of 7-37 month old children from The Gambia shows the development of

2 a distinct non-industrial Prevotella-based trophic network

3

4 Marcus De Goffau ${ }^{1,2 *}$, Amadou T. Jallow ${ }^{3}$, Chilel Sanyang ${ }^{3}$, Andrew M. Prentice 3 , Julian

5 Parkhill 4 , Dora I.A. Pereira $3,5 \$ *$, Josef Wagner ${ }^{1 \# * ~ @ ~}$

$6 \quad *$ These authors contributed equally to the study

7

$8{ }^{1}$ Parasites and Microbes, Wellcome Sanger Institute, Wellcome Genome Campus, Hinxton,

9 Cambridge, CB10 1SA, UK

102 Department of Vascular Medicine, Academic Medical Center, University of Amsterdam,

11 Amsterdam, The Netherlands.

$12{ }^{3}$ Medical Research Council Unit The Gambia at the London School of Hygiene \& Tropical

13 Medicine, Banjul, The Gambia.

$14{ }^{4}$ Department of Veterinary Medicine, University of Cambridge, Madingley Road, Cambridge,

15 CB3 OES, UK

$16{ }^{5}$ Department of Pathology, University of Cambridge, Tennis Court Road, Cambridge CB2 1QP,

17 U.K.

18 \$DIAP has since moved to industry but all work pertaining to this publication was conducted

19 whilst at the University of Cambridge and MRC Unit The Gambia at LSHTM.

20 \#Current address: Victorian Infectious Disease Reference Laboratory, Peter Doherty Institute

21 for Infection and Immunity, Melbourne 3000, Australia

$22 @$ Corresponding authors: Josef Wagner

24 Keywords: African children, Prevotella, microbiome development, trophic network 


\section{ABSTRACT}

26 Distinctive bacterial trophic networks exist in the gut microbiota of individuals in

27 industrialized and non- industrialized countries. To study the development of these networks,

28 we investigated the gut microbiota of 7-37 month old children living in rural Gambia (616

29 children, 1407 stool samples, stratified by 3-month age groups). We found that child age was

30 the largest discriminating factor between samples, and that anthropometric indices WAZ,

$31 \mathrm{HAZ}$, and WHZ, collection timepoints, and iron supplementation did not significantly

32 influence the gut microbiome in this data set. Prevotella copri, Faecalibacterium prausnitzii,

33 and Prevotella stercorea were the most abundant species (35\%, 11\%, and $7 \%$, respectively).

34 Distinct bacterial trophic network clusters were identified, centered around Prevotella

35 stercorea and Faecalibacterium prausnitzii, which were found to develop steadily as the gut

36 microbiome matured. The Prevotella stercorea trophic network cluster is distinct from those

37 found in individuals in industrialized countries and therefore this dataset, set within a critical

38 developmental timeframe, provides an excellent opportunity to understand the influence of

39 a high fiber, low-protein diet on the development of a Prevotella-enriched gut microbiome.

40 
41 Abbreviations:

42 16S V1V2 = bacterial 16S ribosomal RNA variable region 1 and 2

43 CSS = cumulative-sum scaling

44 DNA = deoxyribonucleic acid

45 mths $=$ months

$46 \quad \mathrm{PCoA}=$ Principal Coordinate Analysis

$47 \quad$ TSS $=$ Total Sum Scaling

48 PERMANOVA = PERmutational Multivariant ANalysis Of VAriance

$49 \quad$ PCA $=$ Principal Component Analysis

$50 \quad$ WAZ $=$ Weight-for-Age $Z$ Score

$51 \quad H A Z=$ Height-for-Age Z Score

$52 \quad W H Z=$ Weight-for-Height Z-score

53 FDR = False Discovery Rate (correction method for multiple testing) 
55 One of the largest characterizations of bacterial species present in stool samples to date was

56 from a study from 531 humans representing healthy Amerindians from the Amazonas of

57 Venezuela, residents of rural Malawian communities, and inhabitants of metropolitan areas

58 in the USA ${ }^{1}$. This study was of particular value because it included infants, children,

59 teenagers and adults. Striking differences were observed between USA city residents and

60 rural populations from the Amazonas and Malawi. Species belonging to the genus Prevotella

61 were one of the most dominant differences in the USA versus non-USA comparisons. The

62 importance of Prevotella species as a discriminatory taxon between the gut microbiota of

63 people living in industrialized vs. non-industrialized societies was also highlighted in a study

64 comparing children in West Africa (Burkina Faso) and Europe (Italy) ${ }^{2}$. In this study, the Italian

65 children's gut samples were dominated by Bacteroides and the children's gut samples from

66 Burkina Faso were again dominated by Prevotella.

67 The term enterotype has been proposed to describe distinctive bacteriological patterns of

68 human gut microbiota. Several studies have highlighted distinctive enterotypes ${ }^{3,4}$, one

69 dominated by Bacteroides or Clostridiales species, which is present more commonly in

70 industrialized countries (such as America and Europe) and one dominated by Prevotella

71 species, which is more commonly detected in countries with non-industrialized lifestyles 56 .

72 In the original paper describing enterotypes the human gut microbiome was classified into

73 three groups based on its composition ${ }^{5}$. However, the concept of enterotypes has been

74 critically re-appraised in the last few years. For example, a combined analysis of five studies

75 with 747 samples across five continents could not confirm the enterotypes hypothesis as

76 originally proposed ${ }^{7}$. The same group concluded that the abundances of

77 Prevotella and Bacteroides do not represent consistent microbial communities within the 
"enterotypes", and that "The gut microbiomes of Americans and Europeans having a

Western diet tend to be dominated by Bacteroides and Clostridiales, while rural populations with a high fiber, low-protein diet tend to be dominated by Prevotella". Other groups have also re-analysed the enterotypes hypothesis ${ }^{8,9}$ and these detailed cluster and statistical analyses concluded that only two bacterial communities constitute our gut microbiota, one dominated by Prevotella and one by Bacteroides or species of the Firmicutes ${ }^{9-13}$. In the current study we utilised data from an iron intervention trial in The Gambia in West Africa ${ }^{14}$. Consisting of 616 children, this is the largest paediatric cohort studied thus far in a critical time window for gut microbiome development (7 to 37 months) in a nonindustrialized environment. This exploratory study provides important new insights into the development of trophic networks, including those centred on Prevotella species. Such analyses have to date been mostly performed on Prevotella-poor cohorts from industrialized countries using combinations of adults and children, or to some degree, on smaller paediatric

91 groups in non-industrialized countries ${ }^{15}$.

\section{Study setting for the Iron Intervention Trial in The Gambia in Africa}

93 The study was sponsored by the London School of Hygiene and Tropical Medicine (LSHTM) and conducted at the Medical Research Council (MRC) Unit The Gambia at LSHTM. The Upper River Region (URR) from which the cohort was recruited has an approximate population of 200,000, and only one major town, Basse, and is otherwise typical of rural sub-Saharan Africa. The URR

97 has the highest under-5 mortality rate in the country (92 deaths per 1000 livebirths), the highest percentage of severely malnourished children (7-11\%), and the highest prevalence of malaria and anaemia in children under 5 years ( $4.5 \%$ and $82.5 \%$ respectively) ${ }^{16}$. The study area

100 included 45 villages in the Wuli and Sandu districts, situated in the north bank of the river

101 Gambia, approximately $400 \mathrm{~km}$ east of the capital Banjul, with a population of approximately 
1022800 children aged 6-35 months. All communities have access to borehole tap water at central

103 places. Study specimen samples were collected at one of the study health clinics: Yorrobawol

104 Health Centre, Darsilami Community Health Post, Konkuba Community Health Post, Taibatu

105 Health Post and Chamoi Health Centre.

$106 \quad$ Nutritional and diet information

107 The Gambia is a low-income country in West Africa, where food availability and nutritional

108 status in rural areas are poor. In rural areas, food availability and nutritional status are strongly

109 influenced by seasonality, and a chronically marginal diet is exacerbated by a "hungry season"

110 (July-September), when food stocks from the previous harvest season are depleted. Infants in

111 rural Gambia are breast-fed to 2 years of age, with fewer than half of infants being exclusively

112 breast-fed to 6-months of age as per WHO recommendation ${ }^{17}$. The first foods introduced from

1133 months of age are thin gruels made from only cereal, water (occasionally cow milk is added),

114 salt, and sugar, and are of a low energy and fat content. A thicker porridge made from rice and

115 pounded groundnuts is sometimes administered. Cow milk alone is infrequently given to

116 infants $<1$ year of age; only $57 \%$ of infants receive it more than once a week, although it is

117 provided often to children in the second year of life. From 6 months, infants start to share the

118 family food bowl, the most common meals consisting of boiled rice and a sauce made from

119 groundnuts or leaves. Dried fish may be added to sauces in very small quantities, but fresh fish

120 is not given to infants before 9 months ${ }^{18}$.

121 
RESULTS

\section{Sampling framework and sample characteristics}

124 We performed 16S rDNA amplification and sequencing on a total of 1546 samples of which

125 after quality filtering, oligotyping, contaminant removal, and taxonomic classification, 1407

126 samples from 616 patients were available for detailed analysis. A description of the patient

127 and sampling framework is available in the supplementary Materials and Methods. The

128 samples were collected at three different timepoints during an iron intervention trial in The

129 Gambia ${ }^{14}$ and were used for detailed developmental and bacterial trophic network analysis.

130 The children were initially split into three age groups ( $7-<12$ mths, $12-<24$ mths, and $24-$

13137 mths at the time of enrolment) and subsequently analyzed in detail in 11 age groups. The

132 mean age at enrolment was 22 months ( $7-37$ months) and $48 \%$ of the children were female

133 (Table 1). At the end of study some children were up to 40 months old due to a day 85 stool

134 collection after initial enrolment.

135 Children with severe malnutrition ( $N=88,6 \%$ of children who were screened) ( $\mathrm{z}$-scores for

136 length/height-for-age (HAZ), weight-or-age (WAZ), weight-for-length/height (WHZ) $\leq-3$

137 standard deviations (SD) were excluded from the trial. Mean z-scores for the included

138 children were around -1 (Table 1).

139

140

141

142

143

144

145 
Table 1. Child cohort available for microbiome analysis

\begin{tabular}{|c|c|c|c|c|c|}
\hline \multicolumn{2}{|c|}{ Patient characteristics } & $\begin{array}{l}\text { All age } \\
\text { group }\end{array}$ & $\begin{array}{l}\text { youngest age } \\
7-<12 \text { mths* }\end{array}$ & $\begin{array}{l}\text { middle age } \\
12-<24 \text { mths* }\end{array}$ & $\begin{array}{l}\text { oldest age } \\
24-37 \text { mths* }\end{array}$ \\
\hline \multicolumn{2}{|c|}{ Children number } & 633 & 87 & 266 & 280 \\
\hline \multicolumn{2}{|c|}{ Age min-max (mean) } & $7-37(22)$ & 7-11 (9) & $12-23(17)$ & $24-37(30)$ \\
\hline \multicolumn{2}{|c|}{ Female } & $305(48 \%)$ & $38(44 \%)$ & $139(52 \%)$ & $128(46 \%)$ \\
\hline \multicolumn{2}{|c|}{ Weight (kg), mean (SD) } & $10(1.7)$ & $7.9(1.0)$ & $9.5(6.1)$ & $10.7(2.0)$ \\
\hline \multicolumn{2}{|c|}{ Height (cm), mean (SD) } & $81.6(6.2)$ & $70.7(3.8)$ & $75.85(11.8)$ & $83.3(14.3)$ \\
\hline \multicolumn{2}{|c|}{ WAZ, mean $(S D)(N=1373)$} & $-0.95(0.85)$ & $-0.47(0.88)$ & $-0.94(0.83)$ & $-1.09(0.80)$ \\
\hline \multicolumn{2}{|c|}{$\mathrm{HAZ}$, mean $(\mathrm{SD})(\mathrm{N}=1373)$} & $-0.85(0.94)$ & $-0.38(0.90)$ & $-0.71(0.93)$ & $-1.1(0.89)$ \\
\hline \multicolumn{2}{|c|}{ WHZ, mean $(S D) n(N=1376)$} & $-0.77(0.85)$ & $-0.62(0.90)$ & $-0.88(0.84)$ & $-0.72(0.84)$ \\
\hline \multicolumn{2}{|c|}{ Sample characteristics } & 1407 & 159 & 608 & 640 \\
\hline Timepoint & \begin{tabular}{|l} 
Day 1 \\
Day 15 \\
Day 85
\end{tabular} & $\begin{array}{l}522(37.1 \%) \\
413(29.4 \%) \\
472(33.5 \%)\end{array}$ & $\begin{array}{l}72 \\
55 \\
32\end{array}$ & $\begin{array}{l}238 \\
177 \\
193 \\
\end{array}$ & \begin{tabular}{|l|}
212 \\
181 \\
247 \\
\end{tabular} \\
\hline Treatment & $\begin{array}{l}\text { Treatment } \\
\text { Placebo }\end{array}$ & $\begin{array}{l}950(67.5 \%) \\
457(32.5 \%)\end{array}$ & $\begin{array}{l}106 \\
53 \\
\end{array}$ & $\begin{array}{l}408 \\
200 \\
\end{array}$ & $\begin{array}{l}436 \\
204 \\
\end{array}$ \\
\hline \multirow{5}{*}{$\begin{array}{l}\text { Geographic } \\
\text { location of } \\
\text { health facility }\end{array}$} & Chamoi & $156(11.1 \%)$ & 24 & 86 & 46 \\
\hline & Darsilami & $254(18.1 \%)$ & 28 & 99 & 127 \\
\hline & Kuwonkuba & $133(9.5 \%)$ & 18 & 62 & 53 \\
\hline & Taibatu & $416(29.6 \%)$ & 37 & 163 & 216 \\
\hline & Yorrobawol & $448(31.8 \%)$ & 52 & 198 & 198 \\
\hline
\end{tabular}

147 Abbreviations: min: minimum, max: maximum, mths = months, HAZ: height-for-age $Z$ score, 148 WAZ: weight-for-age Z score, WHZ: weight-for-height Z score, SD: standard deviation

$149 *$ age at enrolment

151 In this large study only 15/633 patients (2.4\%) had received antibiotics within the collection

152 time frame. The reason for receiving antibiotics and the time frame is explained in detail in

153 the online supplementary section 4.8. We performed multivariate PCoA analysis for the

154 middle age group (12 - <24 mths) (Supplementary Figure 1.a) and old age group (24 - 40

155 mths) (Supplementary Figure 1.b) patients comparing the microbiome from the antibiotic

156 treatment samples with the non-antibiotic samples from the day 85 collection time points.

157 PCoA analysis revealed that all antibiotic treatment samples clustered within the expected

158 range of the other samples and did not present as outliers. ANCOM testing ${ }^{19}$ revealed no 
159 significant differences in microbial composition between antibiotic positive and negative

160 samples in the middle age group (data not shown). In the old age group, only one species was

161 statistically significantly depleted in the antibiotic treated group. Holdemanella biformis, with

162 an overall abundance of $0.416 \%$ in all 1407 samples, were detected at a mean abundance of

1630.069 in the antibiotic positive group and at a mean abundance of 0.65 in the antibiotic

164 negative group, Supplementary Figure 1.c (ANOVA uncorrected P value 7.5e-06). As antibiotic

165 use had not caused obvious microbiome composition distortions the analysis was continued

166 with all samples included. The limited or absent effect of antibiotics was most likely due to

167 the large time frame (min 23 days, max 65 days, mean 41 days) between antibiotics being

168 given and the day 85 stool collection.

169

170 
Supplementary Figure 1. Multivariate PCoA did not identify different clustering of patients treated with antibiotics
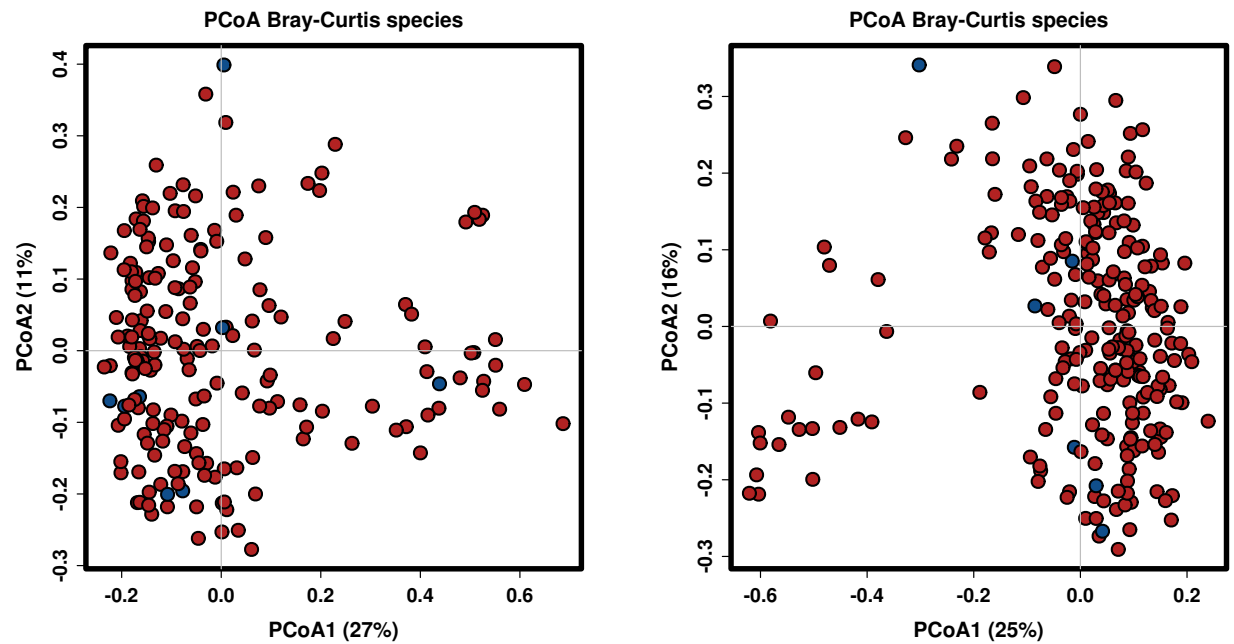

No antibiotic samples

Antibiotic samples

1.c Species difference in the old age groups between children on antibiotics (yes) and non antibiotics (no)

Holdemanella biformis 0.416

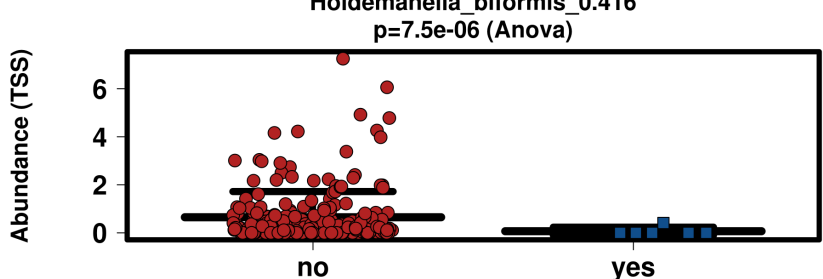

172 Supplementary Figure 1. Multivariate PCoA did not identify different clustering of patients

173 treated with antibiotics

174 The day 85 samples from the middle age group (Sup Fig 1.a) and from the old age group (Sup

175 Fig 1.b) were analysed by PCoA to expose whether children on antibiotics treatment

176 clustered differently from the children without antibiotics. Holdemanella biformis was the

177 only significantly different species between antibiotic treated and non-treated children at the old age group (Sup Fig 1.c)

179

180 
182 As a first step we conducted multivariate and univariate analysis to identify whether children

183 from the treatment arm (iron supplementation) and from the placebo group could be

184 analysed together (Supplementary Figure 2). Multidimensional scaling using Principal

185 Coordinates Analysis (PCOA) did not cluster children differently based on iron supplement

186 treatment or placebo (Supplementary Figure 2.a). Volcano Plot analysis identified only one

187 species which was statistically different between the two sample groups (red dot in

188 Supplementary Figure 2.b). This single species was subsequently identified by the Kruskal-

189 Wallis Rank test as Megamonas funiforms which was detected at higher abundance in the

190 treatment group with a false discovery rate (FDR) corrected P value of 0.0099

191 (Supplementary Figure 2.c). For a second univariate statistical test we used the ALDEx2 test.

192 With the ALDEx2 test no species were identified as statistically significantly different between

193 the treatment and placebo group. The top species was again Megamonas funiforms with a

194 Benjamini Hochberg corrected P value of 0.18 for the Welch's t-test and 0.32 for the

195 Wilcoxon test (Supplementary Figure 2, ALDEx2 table). In addition, a Permutational

196 Multivariate Analysis of Variance (PERMANOA) test did not identify a statistically different

197 beta diversity between the treatment and placebo groups (data not shown).

198

199

200 
Supplementary Figure 2. Multivariate PCoA and univariate statistic did not identify a different microbiome between children on iron supplement treatment and placebo
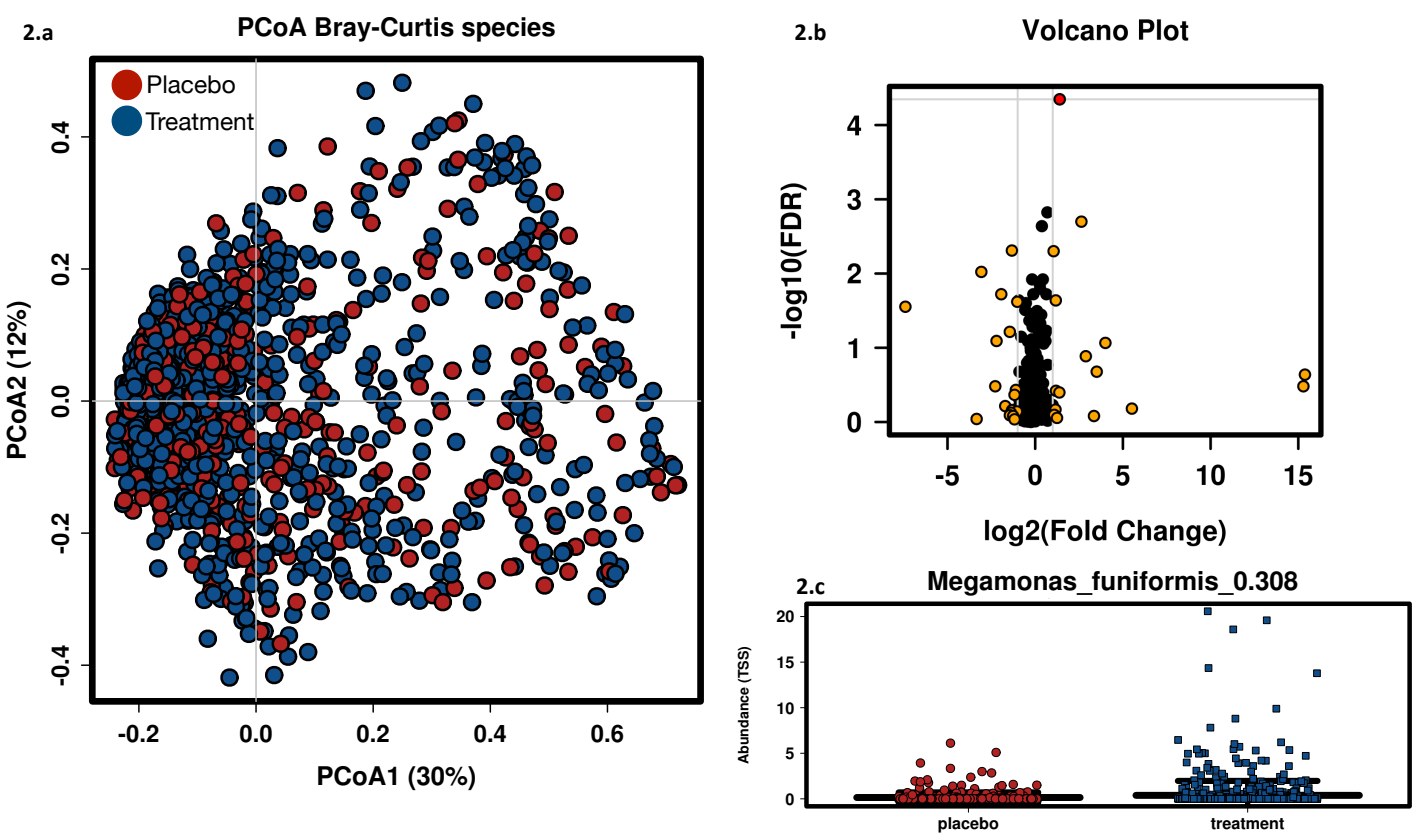

Kruskal-Wallis Rank Test

\begin{tabular}{|c|c|c|c|c|c|c|c|c|}
\hline Taxa $(\mathrm{N}=219)$ & $\mathrm{P}$ (rank test) & Adjusted P (Bonferroni) & FDR & treatment mean & Placebo mean & treatment median & Placebo median & Fold Change \\
\hline Megamonas_funiformis_0.308 & 0.000045 & 0.0099 & 0.0099 & 0.38 & 0.14 & 0 & 0 & 2.635 \\
\hline Phascolarctobacterium_succinatutens_0.284 & 0.0015 & 0.33 & 0.13 & 0.34 & 0.21 & 0 & 0 & 1.617 \\
\hline Lactobacillus_delbrueckii_ 0.016 & 0.002 & 0.44 & 0.13 & 0.022 & 0.0036 & 0 & 0 & 6.211 \\
\hline Coprococcus_sp.HPP0074_0.029 & 0.0023 & 0.5 & 0.13 & 0.033 & 0.025 & 0 & 0 & 1.302 \\
\hline Lactobacillus_mucosae_0.1 & 0.0049 & 1 & 0.18 & 0.065 & 0.16 & 0 & 0 & -2.512 \\
\hline Cetobacterium_somerae_0.152 & 0.005 & 1 & 0.18 & 0.19 & 0.09 & 0 & 0 & 2.07 \\
\hline Lactobacillus_gasseri_0.072 & 0.0095 & 1 & 0.29 & 0.021 & 0.17 & 0 & 0 & -8.397 \\
\hline Erysipelatoclostridium ramosum 0.092 & 0.012 & 1 & 0.29 & 0.1 & 0.075 & 0.005 & 0 & 1.354 \\
\hline Allisonella_histaminiformans_0.421 & 0.012 & 1 & 0.29 & 0.4 & 0.45 & 0.06 & 0.13 & -1.129 \\
\hline 7 & 0.014 & 1 & 0.3 & 0.29 & 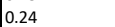 & 4 & 0.1 & \\
\hline
\end{tabular}

ALDEx2 test

\begin{tabular}{|l|l|l|l|l|l|l|}
\hline Taxa (N = 219) & we.ep & we.eBH & wi.ep & wi.eBH & Treatment.median & Placebo.median \\
\hline Megamonas_funiformis_0.308 & 0.003671115 & 0.188101982 & 0.01316173 & 0.320446849 & 0 & 0 \\
Phascolarctobacterium_succinatutens_0.284 & 0.022874477 & 0.33851409 & 0.021533292 & 0.359167159 & 0 & 0 \\
Allisonella_histaminiformans_0.421 & 0.012571362 & 0.341245546 & 0.011244605 & 0.379086281 & 11 & 24 \\
Anaerostipes_sp.5_0.27 & 0.087207799 & 0.568860721 & 0.015656061 & 0.43363021 & 25 & 19 \\
Escherichia_coli_3.505 & 0.084030385 & 0.60753828 & 0.047735245 & 0.533137991 & 125.5 & 174 \\
Lactobacillus_mucosae_0.1 & 0.106601785 & 0.433416708 & 0.154709086 & 0.546906955 & 0 & 0 \\
Lactobacillus_salivarius_0.077 & 0.099588445 & 0.442231554 & 0.142775321 & 0.52718634 & 0 & 0 \\
Lactobacillus_fermentum_0.03 & 0.11175917 & 0.486656866 & 0.13400698 & 0.557791069 & 0 & 0 \\
Coprococcus_sp.HPP0074_0.029 & 0.100757862 & 0.518303646 & 0.058450184 & 0.492780291 & 0 & 0 \\
Weissella confusa 0.097 & 0.06207127 & 0.518328589 & 0.057128628 & 0.522893692 & 0 & 1 \\
\hline
\end{tabular}

Multidimensional scaling using Principal Coordinates Analysis (PCOA) did not cluster children differently based on iron supplement treatment and placebo (Sup fig 2.a). Volcano Plot only identified one species which was statistically different between the two sample groups (red dot in Sup fig 2.b). The only statistically significant species identified by the Kruskal-Wallis Rank test was Megamonas funiforms which was detected at higher abundance in the treatment group with a false discovery rate (FDR) corrected P value of 0.0099 (Sup fig 2.c). For the second univariate statistical test we used the ALDEX2 test. The ALDEx2 test used Bayesian methods to infer technical and statistical error. The ALDEx2 test generated the following output:

we.ep = the expected $\mathrm{P}$ value of the Welch's t-test, we.eBH = the expected value of the Benjamini Hochberg corrected $\mathrm{P}$ value for the Welch's t-test, wi.ep $=$ the expected $\mathrm{P}$ value of the Wilcoxon test, wi.eBH $=$ the expected value of the Benjamini Hochberg corrected $\mathrm{P}$ value of the Wilcoxon test. No taxa were statistically significantly implicated in either of the two sample groups with a Benjamini Hochberg corrected $\mathrm{P}$ value $<0.05$. 
Because of the highly similar bacterial compositions of the treatment and the placebo groups

206 we considered the entire cohort to be highly homogenous and thus all samples were

207 subsequently analysed together as one group. Thus, while this gut microbiome data set does

208 not inform on the case/control aspects of the original study, it does represent an excellent

209 opportunity for understanding the gut microbiome development over time in these African

210 children within this critical gut microbial developmental timeframe.

212 Bacterial changes were observed between the earliest and latest sampling timepoints within

213 individuals.

214 We used Mixed Effect Regression modelling (MERM) to analyze samples from paired

215 repeated timepoints. 682 paired samples were available from 341 patients with a repeated

216 sample collection from D1 and D15, 758 paired samples were available from 379 patients

217 with a repeated sample collection from D1 and D85, and 624 paired samples were available

218 from 312 patients with a repeated sample collection from D15 and D85. (Table 2).

219 Table 2. Longitudinal repeat sample analysis

\begin{tabular}{|c|c|c|c|c|c|c|}
\hline \multirow{2}{*}{$\begin{array}{c}\text { Number of paired samples } \\
\text { (patients) }\end{array}$} & \multirow{2}{*}{$\begin{array}{c}\text { Number of significant species } \\
\text { with FDR P value }<0.05\end{array}$} & \multicolumn{3}{|c|}{ MERM with diversity indexes FDR P value reported } \\
\cline { 4 - 7 } & $758(379)$ & 8 & Richness & Chao1 & Fisher's Alpha & Simpson \\
\hline D1 + D85 & 2 & 0.0017 & $6.00 \mathrm{e}-05$ & 0.0014 & 1 \\
\hline D15 + D85 & $624(312)$ & 0 & 0.15 & 0.0069 & 0.64 & 1 \\
\hline D1 + D15 & $682(341)$ & & 0.29 & 0.056 & 1 & 1 \\
\hline
\end{tabular}

220

221 The largest differences were observed between D1 and D85 sampling timepoints for which

222 eight species were statistically significantly different between the two timepoints with FDR

223 corrected $P$ value $<0.05$. Six of the eight species increased in abundance from D1 to D85.

224 These species and total cohort abundance in \% were Erysipelotrichaceae sp. (0.32\%),

225 Ruminococcaceae sp. (0.23\%), Ruminococcus lactaris (0.09\%), Streptococcus equinus (0.9\%), 
2273 a to 3). Two of the eight species decreased from D1 to D85 with Bifidobacterium being the

228 most abundant species (4.12) followed by Allisonella histaminiformans (0.42\%)

229 (Supplementary Figure $3 \mathbf{g}$ and $\mathrm{h}$ ). The alpha diversity indexes for Richness, Chao1, and

230 Fisher's Alpha were also significantly higher in the D85 samples compare to the D1 samples

231 (Supplementary Figure 3 i to I).

232

233 Significant differences between the young, middle, and old age group samples

234 Beta diversity analysis (between group analysis) was conducted to identify if the microbial

235 composition between the young, middle, and old groups differs from each other. The

236 Bonferroni corrected P-value from the PERMANOVA test between the three different age

237 groups was 0.0003 and the calculated F statistic was between 35 and 125 for the species

238 taxon, between 46 and 163 for the genus taxon, and between 62 and 238 for the family

239 taxon (Supplementary Table 1). For all three taxonomic levels, the F statistic was highest

240 between the young and old age groups.

241

Supplementary Table 1. Pairwise PERMANOVA for taxon species, genera and family with age group variables

\begin{tabular}{|c|c|c|c|c|c|c|c|c|}
\hline \multicolumn{3}{|c|}{ species and age group } & \multicolumn{3}{|c|}{ genus and age group } & \multicolumn{3}{|c|}{ family and age group } \\
\hline Bonferroni corrected $\mathrm{P}$ value & Young & Middle & Bonferroni corrected $\mathrm{P}$ value & Young & $\begin{array}{l}\text { Middle } \\
\end{array}$ & Bonferroni corrected $\mathrm{P}$ value & Young & Middle \\
\hline Old & 0.0003 & 0.0003 & Old & 0.0003 & 0.0003 & Old & 0.0003 & 0.0003 \\
\hline Young & & 0.0003 & Young & & 0.0003 & Young & & 0.0003 \\
\hline F statistic & Young & Middle & F statistic & Young & Middle & F statistic & Old & Middle \\
\hline Old & 125.3 & 35.28 & Old & 163.3 & 46.05 & Old & 237.6 & 84.54 \\
\hline Young & & 53.11 & Young & & 65.01 & Young & & 62.27 \\
\hline
\end{tabular}

242 Supplementary Table 1. Pairwise PERMANOVA for taxon species, genera and family with age

243 group variables for young, middle, and old age groups

245 The PERMANOA test-confirmed differences between young, middle, and old age were also

246 analysed using PCOA which shows a different microbial population between each of the three 
age groups at the species (Figure 1.a), genus (Figure 1.b), and family (Figure 1.c) level. As

248 expected, the young and old age groups were separated the furthest apart from one another

249 while the middle age group clustered between them. It also showed that the greatest

250 separation was observed at the lowest (species) taxonomic resolution. We also plotted all

251 species, genera, and families with a minimum abundance of $0.5 \%$ across all samples to show

252 which taxa were most responsible for the separation. At the species level, these included

$25322 / 491$ species encompassing 82\% of all bacterial 165 reads (Figure 1.a). At the genus level

254 these included 26/216 genera which included $89 \%$ of all reads (Figure $1 . b$ ) and at the family

255 level these include 18/71 families which included $97 \%$ of all reads (Figure 1.c). In our analysis,

256 age was the only contributing factor which differentiated the gut microbiomes of samples in

257 this cohort. Further detailed analysis was therefore conducted to investigate the maturation

258 of the gut microbiome in this large rural community from The Gambia in West Africa during

259 the first three years of life based on age, split into 11 age groups (3-month intervals). 
芒芯䒘
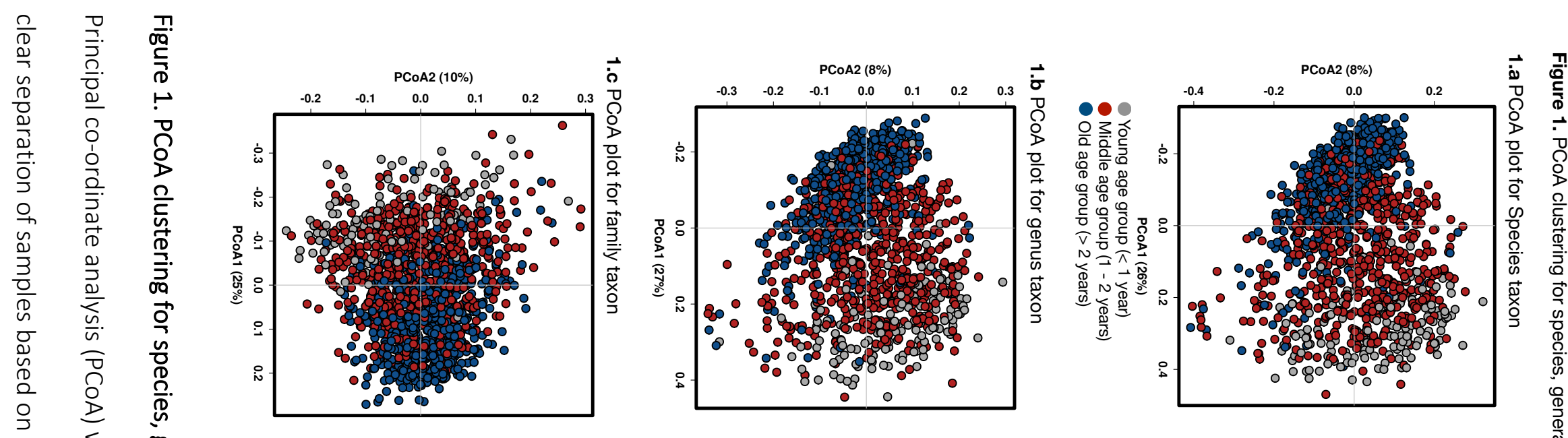

elative abundance
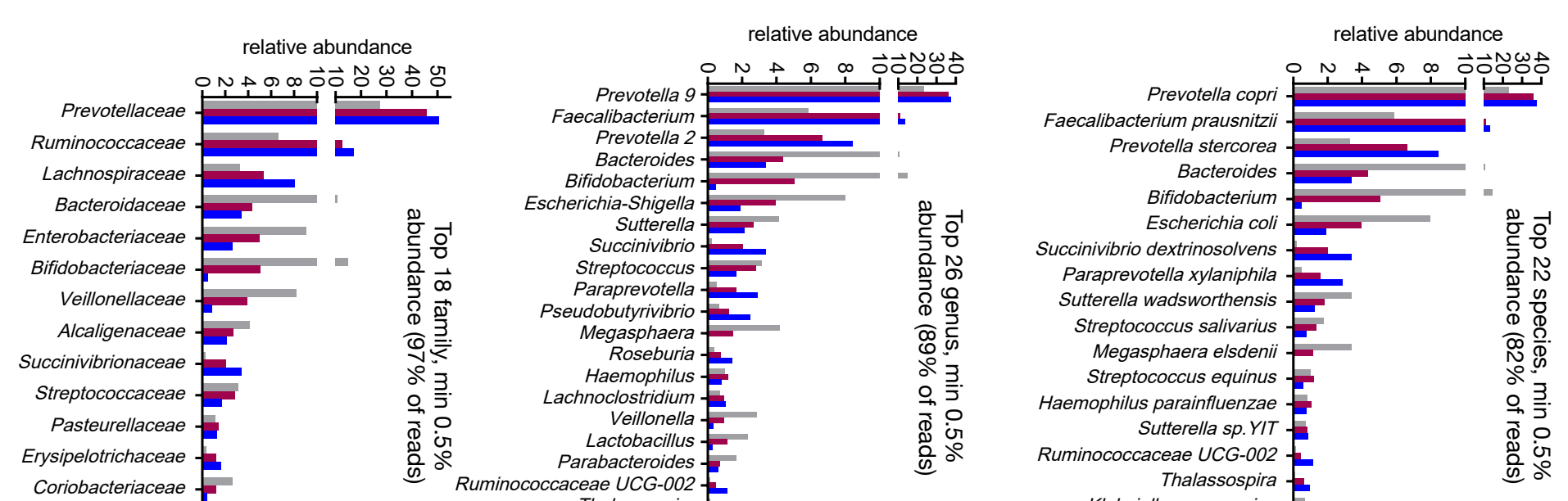

等 Bacteroides Escherichia-Shigella

Sutterella
Succinivibrio

Streptococcus

Pseudobutyrivibrio

Megasphaera

Roseburia
Haemophilus

Lachnoclostridium -

Veillonella
Lactobacillus

Parabacteroides

Porphyromonadaceae

Lactobacillaceae

Fusobacteriaceae -

Rhodospirillaceae

Thalassospira $F$

Collinsella

Fusobacterium

Prevotella 1.

Prevotella

Escherichia coll

Parapreo dextrinosolvens

Sutterela walla xylaniphila

Streptococcus salivarius

Megasphaera elsdenii

Streptococcus equinus

Haemophilus parainfluenzae -

Sutterella sp.YIT

Ruminococcaceae UCG-002

Thalassospira -

Klebsiella pneumoniae

Eubacterium rectale

1]

음 흥.

을 흥. 음

Fusobacterium mortiferum -

Prevotella ruminicola

Pseudobutyivbrio ruminis -

Lactobacillus ruminis

1

음 콩. 을 
265 1.a), the genus taxonomic level (Figure 1.b), and the family taxonomic level (Figure 1.c). The

266 column graphs next to the PCoA plots show the relative abundance of the top species with a

267 minimum abundance of $0.5 \%$ for the species taxon $(n=22)$, for the genus taxon $(n=26)$, and

268 for the family taxon ( $n=18)$. All Bacteroides species and all Bifidobacterium species were

269 combined.

270

271

272 Significant changes in the bacterial alpha diversity over time from 7 to 40 months of age

273 To identify changes over time in the whole data set, we split the data into 11 age groups,

274 separated by three-month intervals, and analyzed alpha diversity. The Fisher's alpha

275 parameter indicated a statistically significant increase in alpha diversity from the youngest

276 age group (7-9 months) to the $34-36$ months age group (Kruskal-Wallis $\mathrm{P}<0.0001$ ) (Figure

277 2.a). The Simpson's index was also statistically significantly different between the 11 age

278 groups, but an upwards trend as seen by the Fisher's alpha was not observed (Kruskal-Wallis

$279 P=0.0002$ ) (Figure 2.b). Both the richness estimator index Chao1 (Figure 2.c) and the

280 observed richness (Figure 2.d) increased over time and this increase was highly significant for

281 both tests (Kruskal-Wallis $\mathrm{P}<0.0001$ for both).

282

283

284 
Figure 2. Alpha diversity indexes for 11 age groups
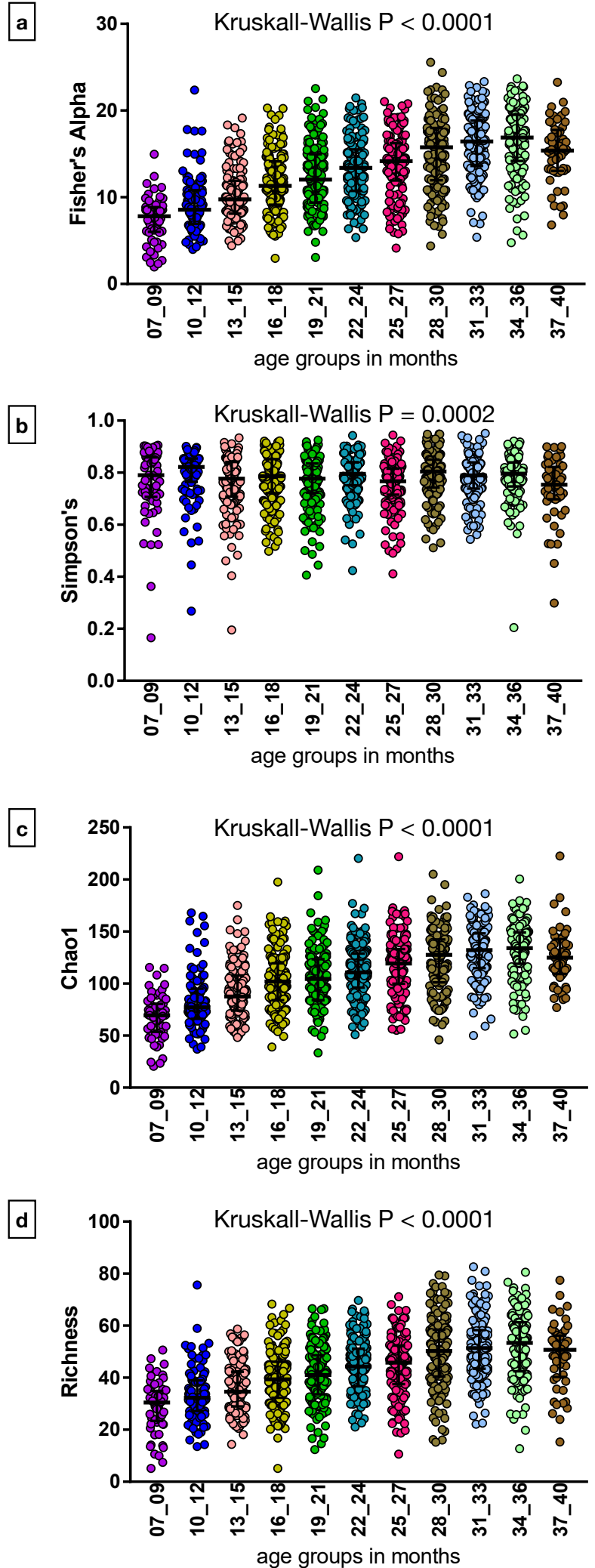
287 Alpha diversity was measured by two different diversity indexes and by two different richness

288 indexes. Figure 2.a The Fisher's alpha diversity test, Figure 2.b Simpson's diversity test, Figure 2.c, Chao1 estimated richness, Figure 2.d Observed richness

292 Maturation of the gut microbiome in a rural community from The Gambia during the first three years of life

294 We used the non-parametric Kruskal-Wallis rank test to identify significantly differentially abundant bacterial taxa in the 11 age groups (3-month intervals), (Supplementary Table 2). From the Kruskal-Wallis rank test we report the uncorrected and the false discovery rate 297 (FDR) corrected P value, as well as the mean and median abundancies for the different taxa. In the 11-age-group comparison 301/488 taxa were statistically different between the age

299 groups of which a large majority (231/301) had a low abundance $(<0.1 \%)$. The top 70 significantly different species with a minimum abundance of $0.1 \%$ include $92.5 \%$ of all

301 sequence reads. Prevotella copri was the most abundant species, with an average abundance 302 in all samples of $35.2 \%$ of all reads. The second and third most abundant species were

303 Faecalibacterium prausnitzii (11.4\%) and Prevotella stercorea (7.1\%), respectively. These

304 three species accounted for a total of $53.6 \%$ of all reads (Supplementary Tables 2 , first three 305 species entries in column "Taxa"). All Prevotella species combined accounted for $27 \%, 44 \%$, 306 and $48 \%$ of all reads, in the young, middle, and old age groups respectively.

307 The maturation pattern over time of the top ten bacterial taxa, shown either at the species or 308 genus level, with a minimum abundance of $1 \%$, which covers $73.2 \%$ of all bacterial $16 \mathrm{~S}$ reads, 309 are shown in Figure 3. These ten bacterial groups are from four major bacterial phyla namely, 
310 Bacteroidetes; Prevotella copri (35.2\%), Prevotella stercorea (7.6\%), Bacteroides (4.6\%),

311 Paraprevotella xylaniphila (2\%); Firmicutes; Faecalibacterium prausnitzii (11.4\%),

312 Streptococcus salivarius (1.1\%); Actinobacteria; Bifidobacterium (4.1\%); and Proteobacteria;

313 Escherichia coli (3.5\%), Succinivibrio dextrinosolvens (2.4\%), Sutterella wadsworthensis (1.8\%).

314 The maturation pattern was investigated with two different normalized datasets.

315 The abundance of Faecalibacterium prausnitzii, Prevotella stercorea, Succinivibrio

316 dextrinosolvens, and Paraprevotella xylaniphila increased steadily over time which can be

317 seen clearly in the 11 age groups dataset. However, Bacteroides, Bifidobacterium, Escherichia

318 coli, Sutterella wadsworthensis, and Streptococcus salivarius show the reverse pattern.

319 Prevotella copri, the most abundant bacterium, reached a very high abundance level ( $\sim 0 \%$

320 soon after the age of 12 months and maintained its dominance after that. An interesting

321 observation was the very far from normal distribution within development periods of

322 Prevotella stercorea, Succinivibrio dextrinosolvens and Paraprevotella xylaniphila. These

323 species co-developed in a distanced bivariate distribution compared to the other dominant

324 species presented in Figure 3, suggesting separate development of high-and low-abundance

325 populations. These 3 species showed a fast increase in abundance up to $18^{\text {th }}$ months of age,

326 followed by a steady state and then again, an increase in abundance between the age of 30

327 months and 36 months followed with a slight drop in abundance after 3 years of age. 
Figure 3. Abundance of the top 10 species with a minimum abundance of $1 \%$ over time
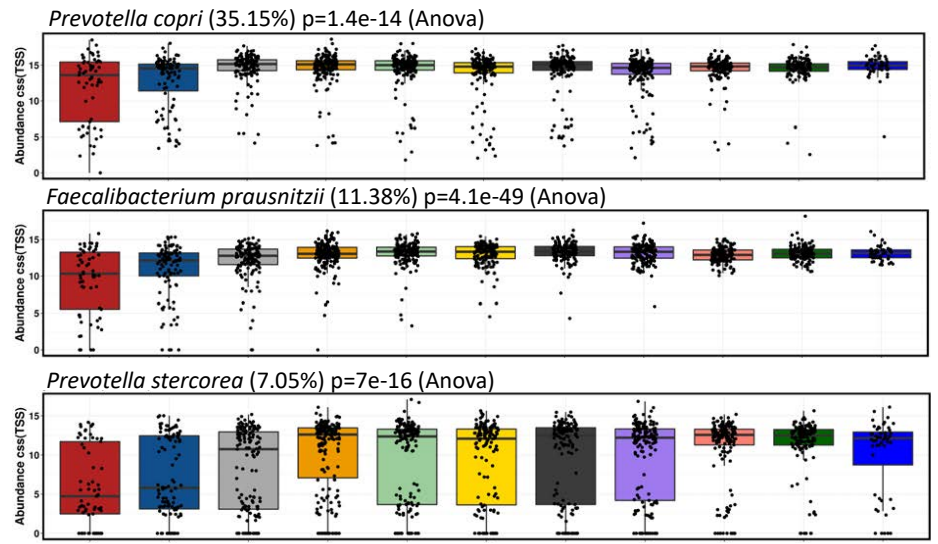

Bacteroides (4.64\%) $\mathrm{p}=1.3 \mathrm{e}-11$ (Anova)
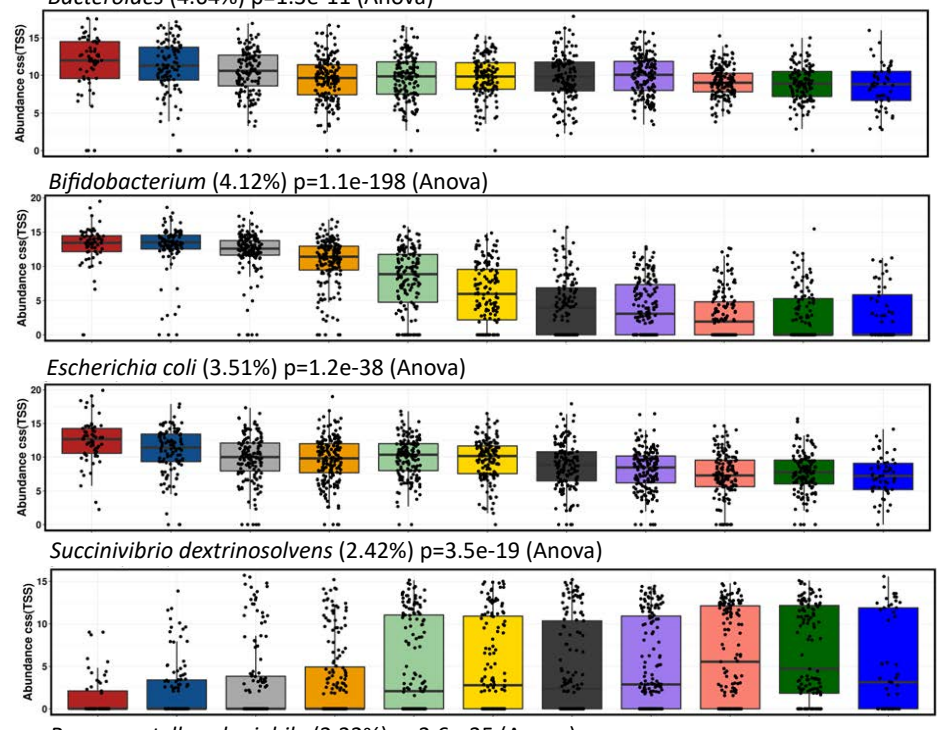

Paraprevotella xylaniphila (2.22\%) $\mathrm{p}=2.6 \mathrm{e}-35$ (Anova)
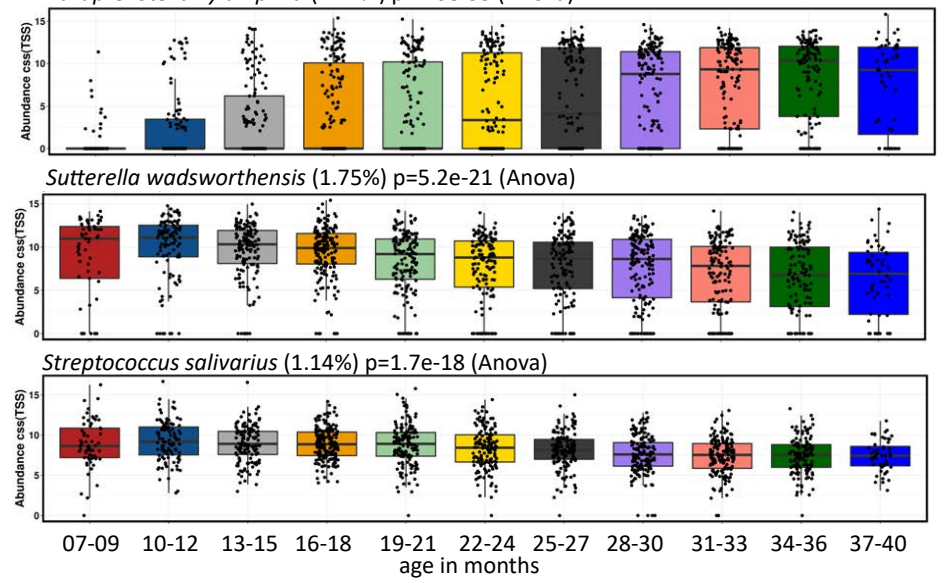

330 Figure 3 . Top 10 species with a minimum abundance of $1 \%$, significantly associated in the 11 
332 Figure 3.a shows the significantly differentially abundant bacterial taxa using relative (total

333 sum scaling (TSS) abundance. Figure 3.b shows the same dataset further transformed using

334 cumulative sum scaling (CSS) +log 2 transformation.

335

336

337 A direct comparison of taxa negatively and positively associated with age is presented in

338 Figure 4. Figure 4.a shows the top 11 negatively associated taxa with age (Spearman's rho

$339 \min -0.3$ and min abundance of $0.1 \%$ ) and Figure 4.b shows the top 20 positively associated

340 taxa with age (Spearman's rho min 0.3 and min abundance of 0.1\%).The largest upward and

341 downward shifts occurred within the first $\sim 26$ months of life.

342

343

344

345 
Figure 4. Top significant taxa associated with age development between the age of 7 months and 40 months

4.a Negative correlated taxa with age with a Spearman Rho index of min $-0.3(-0.3$ to -0.69$)$ and a Spearman FDR $P<0.05$ and a minimum abundance of $0.1 \%(\mathrm{~N}=11$ taxa)

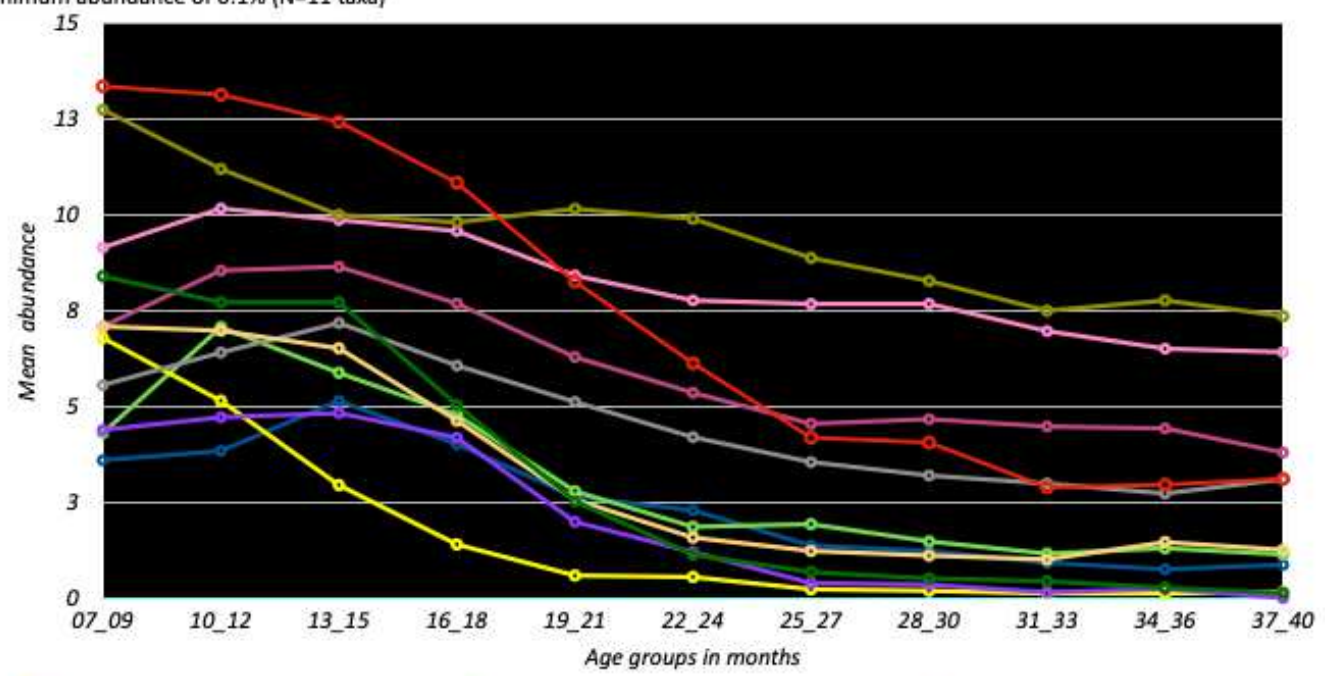
- Bifidabacterium $(4.12 \%$
- Megasphaera elsdenii (0.9\%)
- Collinsella aerofaciens (0.4\%)
Veillonella ratti $(0.3 \%)$ Lactobacillus ruminis $(0.54 \%$
- Megamonas funiformis $(0.31 \%)$
- Sutterella wadsworthensis (1.75\%)
Olsenella sp. SK9K4 (0.24\%)
Escherichia coll (3.51\%

4.b Positive correlated with age with a Spearman Rho index of $\min 0.3(0.3$ to 0.56$)$ and a Spearman FDR $P<0.05$ and a minimum abundance of $0.1 \%(\mathrm{~N}=20)$

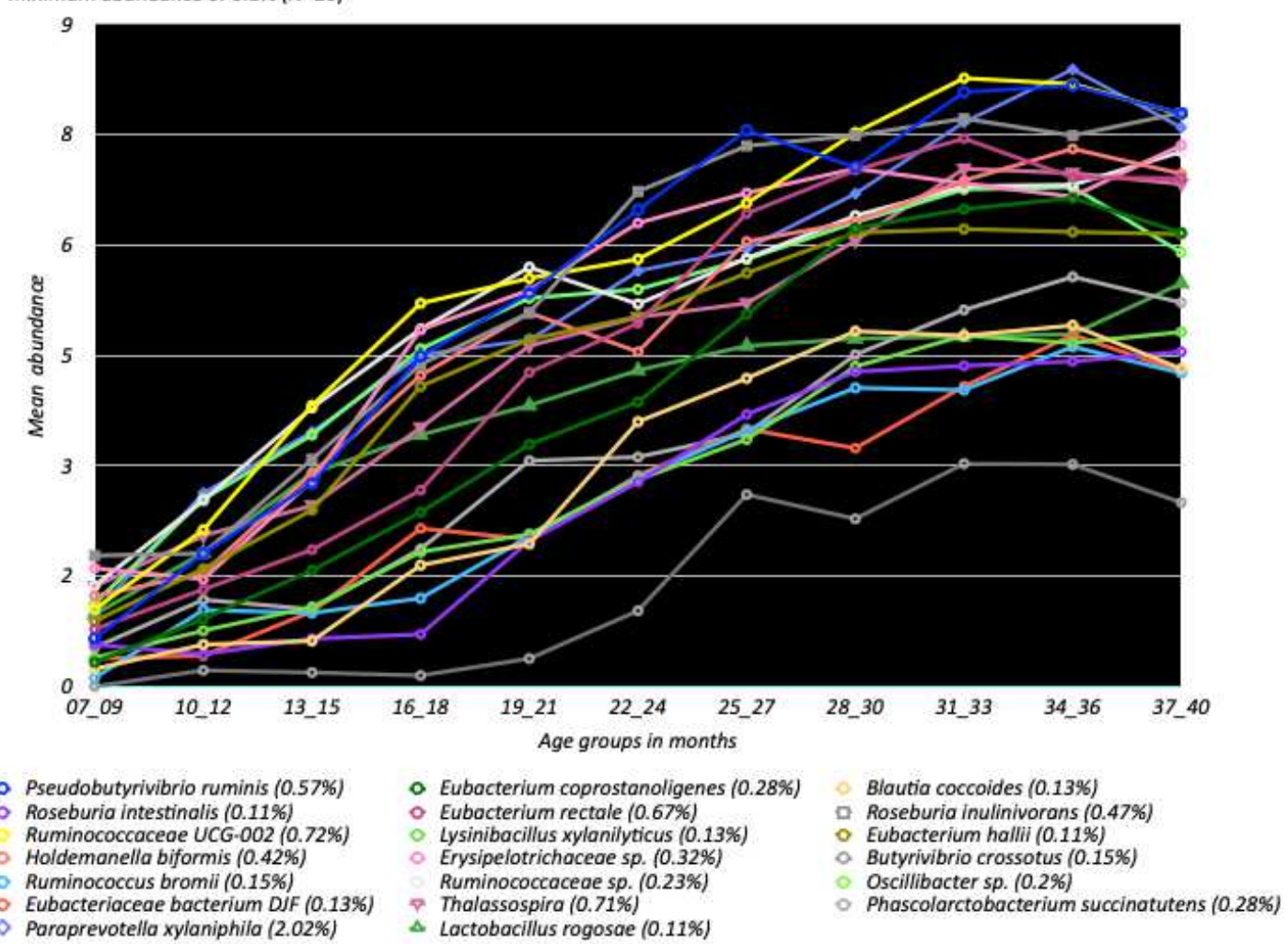

347 Figure 4. Top significant taxa associated with age development between the age of 7 months 
349 All taxa $(\mathrm{N}=11)$ associated with a decreasing abundance over time with a minimum

350 Spearman's rho index of -0.3 from the minimum $0.1 \%$ abundance taxa dataset are presented

351 in Figure 4.a. And, all taxa $(\mathrm{N}=20)$ associated with an increasing abundance over time with a

352 minimum Spearman's rho index of 0.3 from the minimum $0.1 \%$ abundance taxa dataset are

353 presented in Figure 4.b.

356 Correlation analysis identifies distinct clusters representing bacterial trophic networks

357 Microbial populations form clusters based on nutrients available ${ }^{20}$, i.e., at the trophic level a

358 microbial population constitutes a food web of metabolically interdependent organisms ${ }^{21}$.

359 To identify these trophic networks, we first generated a heatmap of the Spearman Rho

360 correlation coefficients between the different bacterial species including the top 51 species

361 with a minimum abundance of $0.2 \%$ in all samples to visualize distinct clusters of bacteria

362 (Figure 5.a). The most distinct and visually striking cluster that was identified was the cluster,

363 indicated with a green box, that includes the third most dominant species, Prevotella

364 stercorea, and consists of species generally not found in the microbiota of individuals from

365 industrialised countries. Succinivibrio dextrinosolvens and Paraprevotella xylaniphila, which

366 showed the same non-normal distribution development pattern as $P$. stercorea (Figure 3) are

367 part of this cluster, highlighting the codependence of these species. A second cluster,

368 indicated with a light blue box, which contains many species found in typically industrialised

369 microbiome compositions, also contained the second most abundant species,

370 Faecalibacterium prausnitzii. A third apparent cluster, indicated in black and containing the

371 Bifidobacterium genus, is the result of species associating with one another due to being

372 prevalent mainly or only within young subjects. Finally, a cluster of bacteria typically found in 
373 the small intestine (like Veillonella dispar and Streptococcus salivarius) is indicated with a

374 purple box. The most dominant species, P. copri (* in heatmap Figure 5.a), was not strongly

375 associated with any of the clusters but is clearly negatively correlated with Bacteroides (** in

376 heatmap Figure 5.a). A summary of the correlations between the main groups of organisms

377 found in this study is shown in Figure 5.b.

378 The green box (Prevotella stercorea \& co) and the blue box (Faecalibacterium prausnitzii \&

379 co) most likely represent trophic networks of interdependent species which develop steadily

380 over time at cluster specific rates. The F. prausnitzii \& co cluster takes about 2 years to

381 mature and reach a stable level (Supplementary Figure 5.a) while the $P$. stercorea \& co

382 cluster takes about 2.5 years to reach a stable state (Supplementary Figure 5.a). Prevotella

383 copri on the other hand is not dependent on any other bacteria as it rapidly becomes the

384 dominant species within 12 months (Figure 3.a and Supplementary Figure 4).

385

386 
Supplementary Figure 4. Development of bacterial trophic network species in 11 age groups (three-months interval)

4.a Development of bacterial trophic network in the Prevotella stercorea cluster together with Prevotella copri

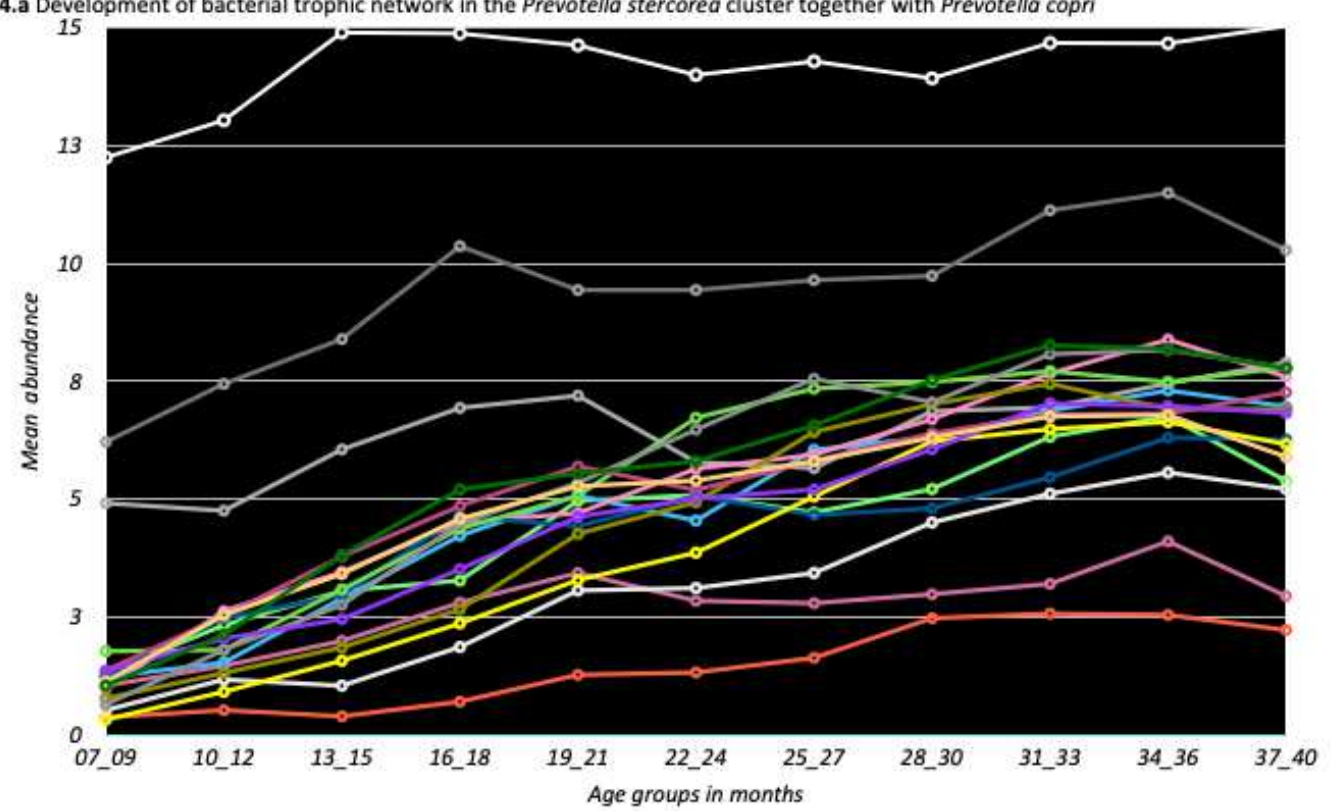

Prevotella copri (35.153\%)

- Thalassospira (0.707\%)

Eubacterium coprostanoligenes

Holdemanella biformis $(0.416 \%$

- Pasteurella multocida (0.249\%)
- Ruminococcaceae UCG.002 (0.716\%)

- Ruminococcaceae sp. (0.231\%)

- Roseburia inulinivorans $(0.465 \%)$

Paraprevotella xylaniphila (2.02\%)

Phascolarctobacterium succinatutens $(0.284 \%)$

- Catenibacterium mitsuokai (0.294\%)
Oscillibacter sp. (0.202\%)

- Pseudobutyrivibrio ruminis $(0.568 \%)$

- Eubacterium rectale (0.673\%)

- Prevotella stercorea (7.054\%)

Succinivibrio dextrinosolvens (2.424\%)

Sutterella sp. (0.82\%)

4.b Development of bacterial trophic network in the Faecalibacterium prausnitzii cluster together with Prevotella copri

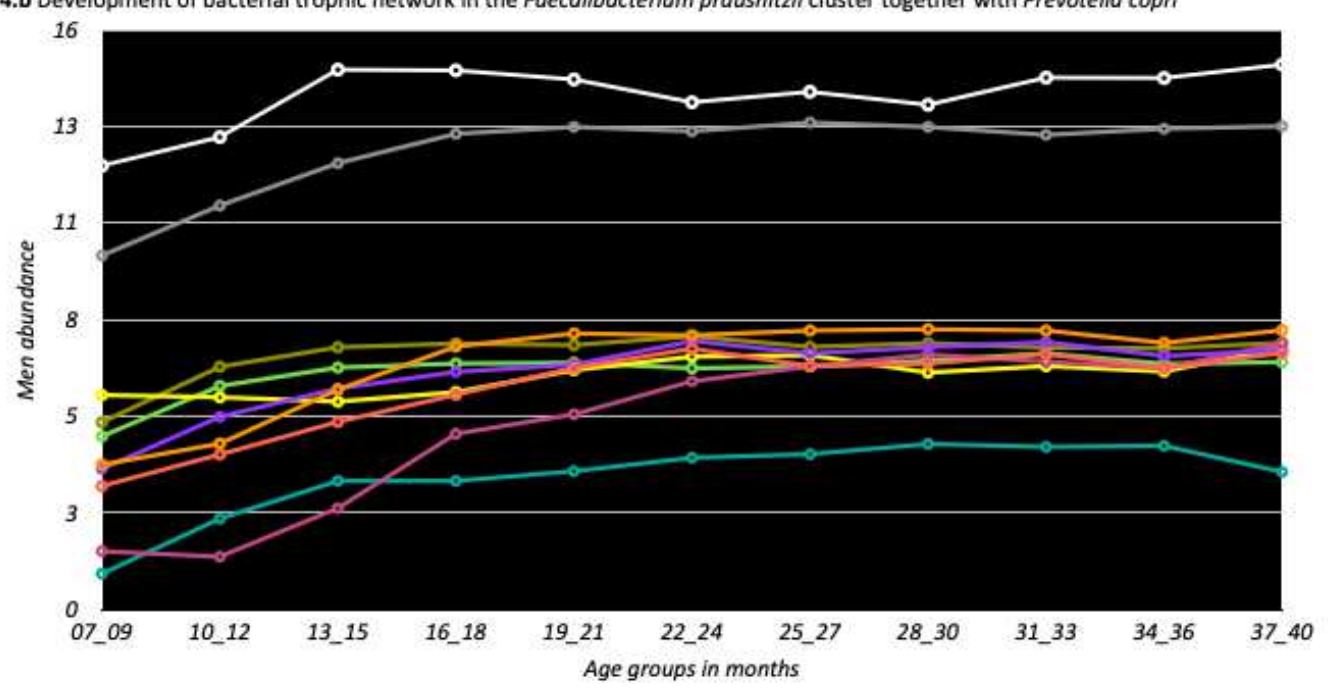

Prevotella copri (35.153\%)

Lachnoclostridium sp. (0.215\%)

Ruminococcus gnavus (0.311\%) O Dorea sp. $(0.214 \%)$

- Anaerostipes sp.5 (0.27\%)

Roseburia faecis $(0.213 \%)$

Dorea sp. (0.214\%)

prausnitziil (11.383\%)

\section{Supplementary Figure 4. Development of bacterial trophic network species over time}

389 The mean abundances of the individual trophic network species are shown as a stacked line

390 graph for the Prevotella stercorea network (Sup Fig 4.a) and for the Faecalibacterium 
prausnitzii network (Sub Fig 4.b) for the 11 three- months age groups. The mean abundances

392 in \% were calculated from total sum scaling (TSS) normalised absolute read counts which

393 were subsequently transformed using cumulative sum scaling (CSS)+log2.

394

395

396 However, the Faecalibacterium \& co. cluster does seem to benefit from a high abundance of

397 P. copri (Figure 6.a) whilst the Prevotella stercorea \& co. cluster does not seem to have a

398 distinct interaction with P. copri (Figure 6.b). Several of the species associated with the

399 Prevotella stercorea \& co. and the Faecalibacterium \& co. clusters seem to be shared

400 between both clusters to some degree, having high positive correlation coefficients with

401 members from both clusters (green and blue clusters in Figure 5.a and b).

402 The steady build-up of $F$. prausnitzii cluster over time appears to mirror the development of a

403 trophic network seen in children in industrialised counties ${ }^{15}$, where the build-up of a trophic

404 network of fibre degraders, acetate and lactate producers combined with butyrate producers

405 appears to be essential for a healthy immune system development. However, the addition or

406 expansion of the P. stercorea \& co trophic network in our African cohort (alongside P. copri)

407 likely helps explain why higher levels of short-chain-fatty-acid production levels are seen in

408 Africans with non-industrialized gut microbiomes. The apparent positive association of

409 Bifidobacterium with various other bacteria (black box, Figure 5.a) is most likely an indirect

410 effect caused by being abundant in the young age group. These positive associations do not

411 seem to represent a trophic network except possibly for the association of Bifidobacterium

412 (which produces lactate) with Megasphaera elsdenii (which metabolizes lactate and produces

413 butyrate). We also note that Megasphaera micronuciformis is positively correlated with

414 Bifidobacterium (Figure 5.a). 
Figure 5. Species association and trophic networks

5.a Heatmap analysis for trophic networks of the top 51 species with minimum $0.2 \%$ abundance
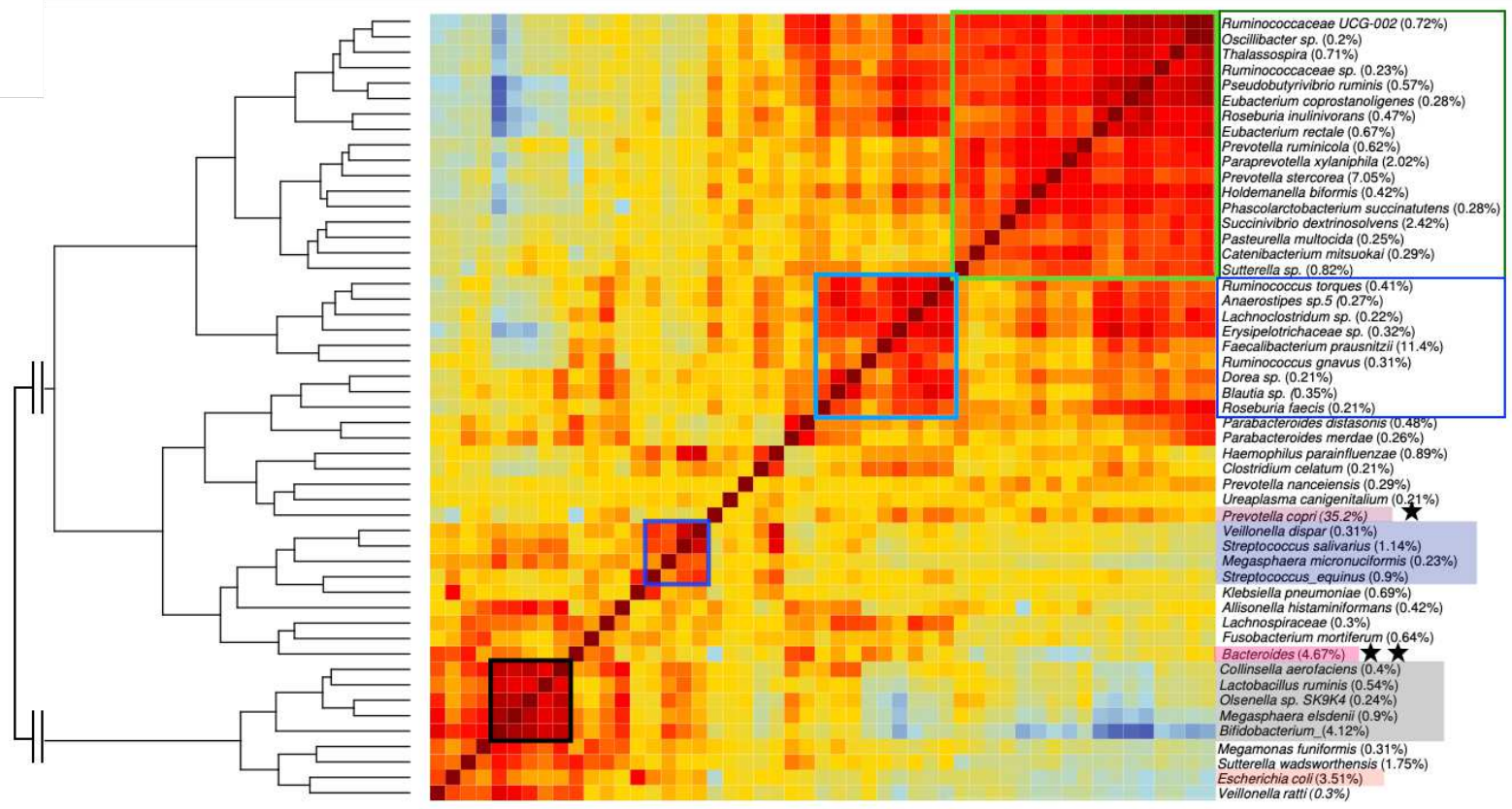

5.b Bacterial network - Summary

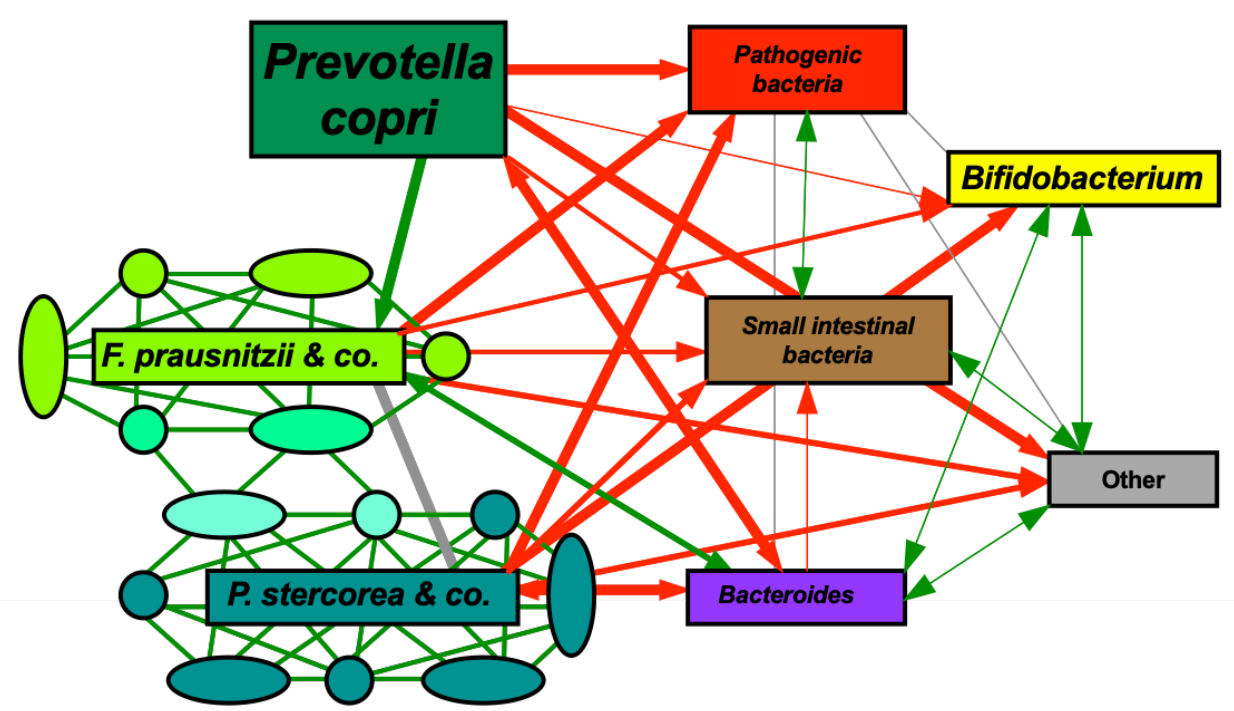

416

417 Figure 5. Species association and trophic networks

418 Figure 5.a A network heatmap was generated using the top 51 species with a minimum

419 abundance of $0.2 \%$ in all samples. The most dominant clusters identified in the bacterial

420 trophic network correlation analysis are highlighted by different coloured boxes. The 
421 Prevotella stercorea network is highlighted in green, the Faecalibacterium prausnitzii network

422 in blue, the Bifidobacterium network in grey, and the small intestinal microbiome network in

423 purple. The red heatmap colour indicates strong positive correlation and the blue heatmap

424 colour indicates strong negative correlation. There are additional species correlated outside

425 the obvious labelled clusters, which are labelled by lines. A bacterial network summary is

426 presented in Figure 5.b. Spearman correlation coefficient analysis was used to identify a

427 bacterial trophic network with the strongest self-correlations. The leading species in each

428 network is highlighted. A positive correlation is highlighted by green lines, a negative

429 correlation is highlighted by red lines, and intermediate correlation is highlighted by grey

$430 \quad$ lines

431

432

433 Principal Component Analysis (PCA) combined with Spearman rho correlation analysis was

434 performed to analyse the maturation process in more detail. Principal component 1 (PC1)

435 represents the maturation process as the accumulation of Prevotella copri and both the $F$.

436 prausnitzii and P. stercorea trophic networks over time vs. the decline of the Bacteroides

437 genus. The build-up of the trophic networks over time is furthermore especially well

438 represented by PC3 (Figure 6.d). PC2 mainly demonstrates how P. copri and the P. stercorea

439 network are negatively correlated with Bacteroides whilst the F. prausnitzii network is

440 positively correlated with Bacteroides (Figure 6.e). PC4 highlights that $P$. copri and the $F$.

441 prausnitzii network are positively correlated with one another (Figure 6.f) but that this

442 positive correlation does not extend to the Prevotella stercorea network. 
Figure 6. Maturation process analysed by Principle Component Analysis combined with Spearman rho correlation
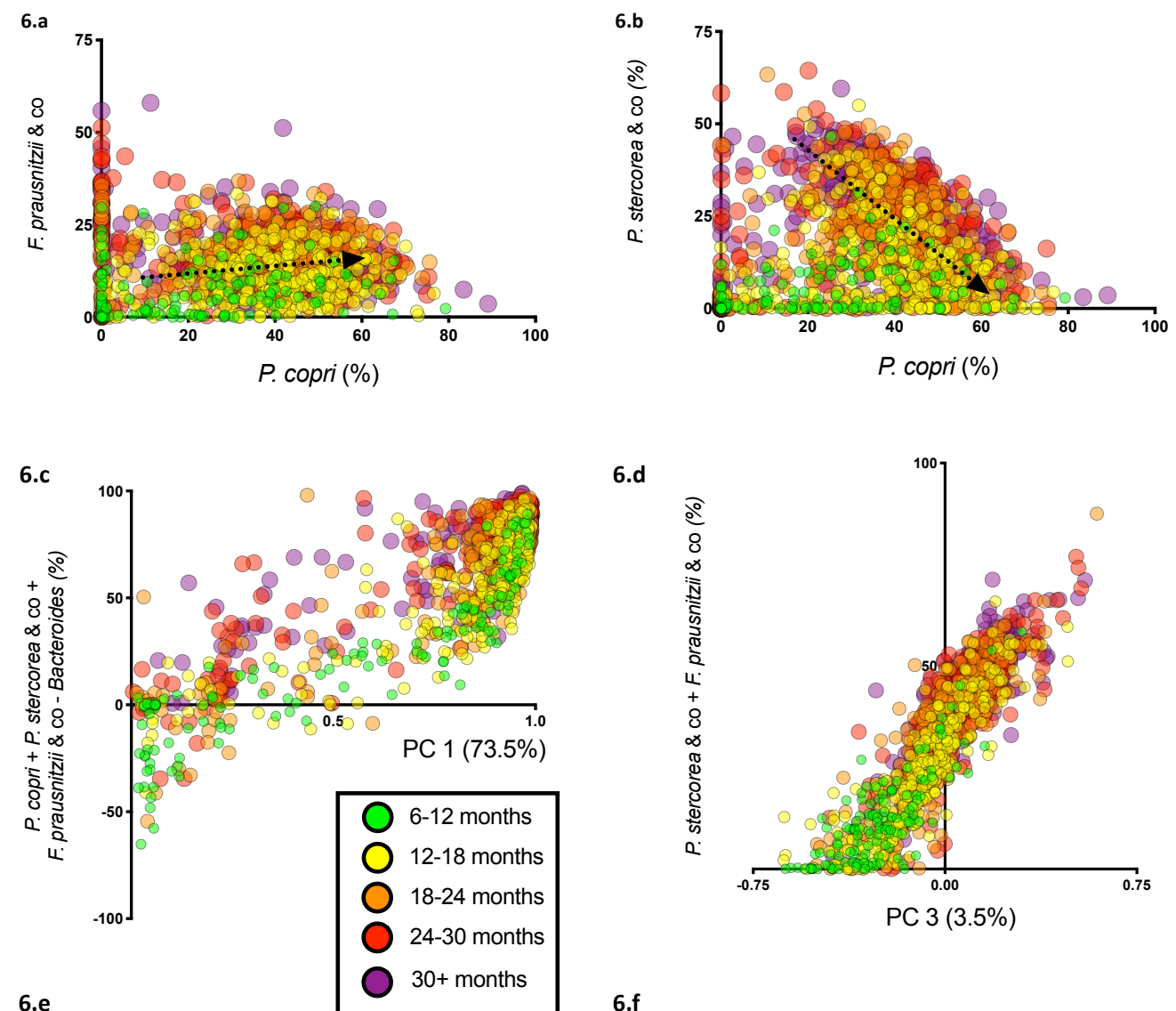

6.d
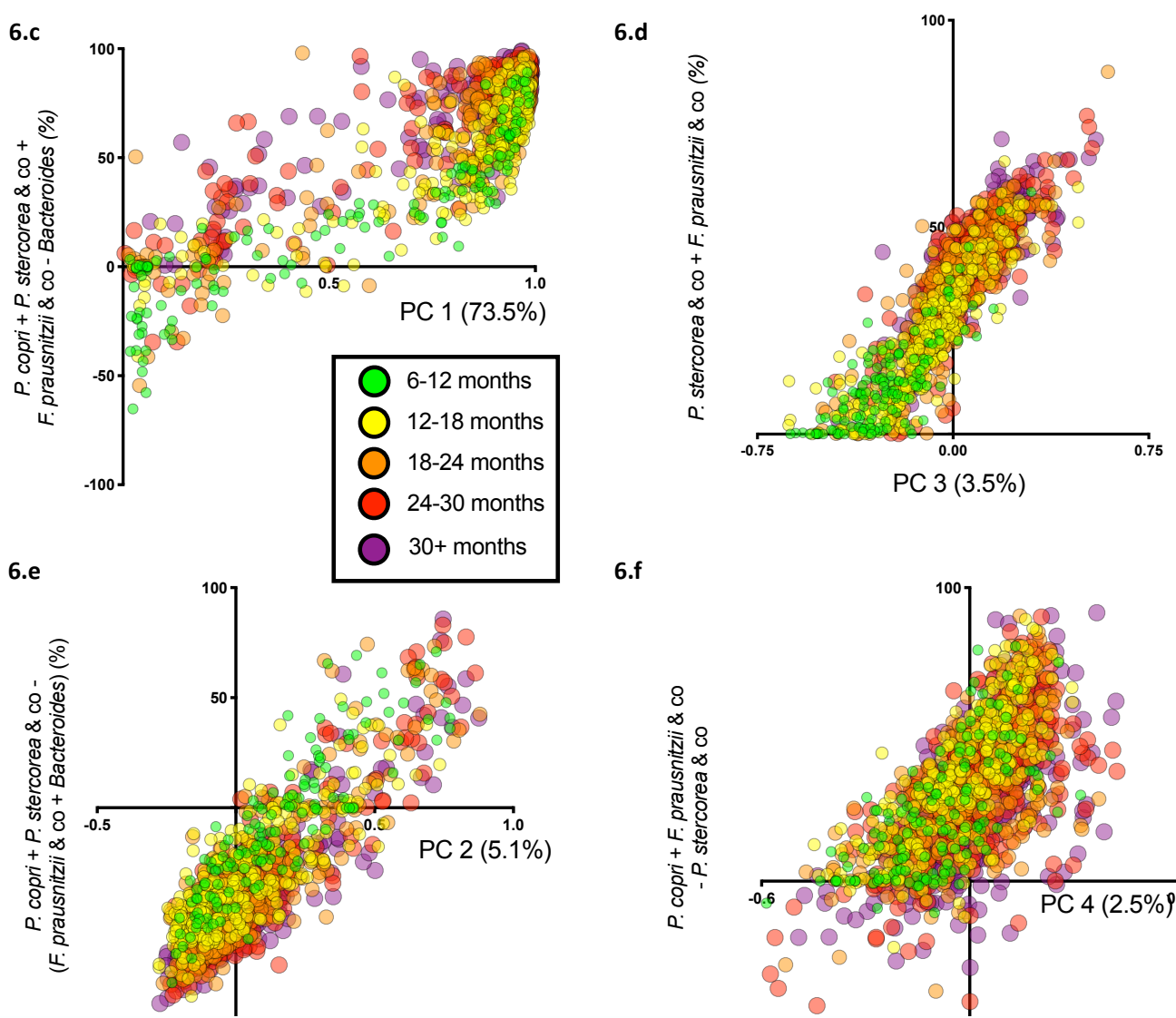

$6 . f$

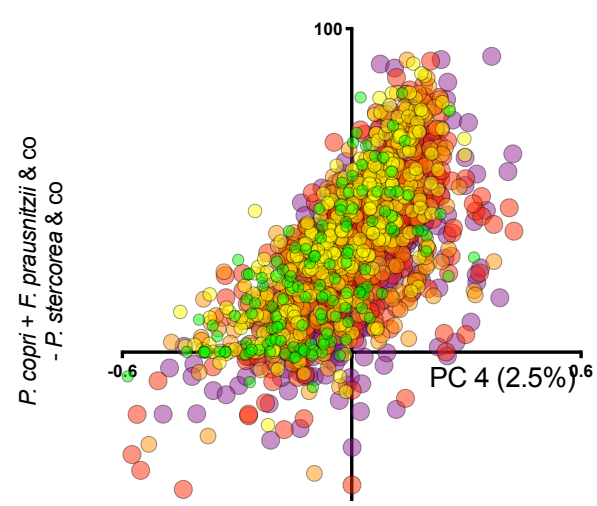

443

444 Figure 6. Maturation process analysed by Principal Component Analysis combined with

\section{Spearman rho correlation}

446 A Principal Component Analysis (PCA) combined with Spearman rho correlation analysis was

447 performed to analyse the maturation process in more detail for different dominant taxa and

448 bacterial networks. The correlations between F. prausnitzii and P. copri are shown in Figure 
449 6.a. Figure 6.b shows the correlation between P. copri and P. stercorea \& co cluster. Principal 450 component 1 (PC1) in Figure 6.c represents $73.5 \%$ of the variation in the data and indicates

451 the maturation process to be the accumulation of Prevotella copri and both the F. prausnitzii 452 and $P$. stercorea trophic networks over time. The build-up of the trophic networks over time

453 is furthermore well described by PC3 in Figure 6.d. PC2 in Figure 6.e indicates how P. copri 454 and the $P$. stercorea network are negatively correlated with Bacteroides whilst the $F$. 455 prausnitzii network is positively correlated with Bacteroides. PC4 in Figure 6.f indicates that $P$. 456 copri and the F. prausnitzii network are positively correlated with one another

(HAZ), and Weight-for-Height z-score (WHZ)

Our study population in terms of WAZ, HAZ, and WHZ had a mean Z score of $-0.95,-0.85$, and

(Table 1). In order to investigate whether different bacterial taxa were associated with these three $Z$ scores values we used multiple linear regression (MLR) for the identification of an association between bacterial taxa and the clinical factors WAZ, HAZ, and WHZ. The association between clinical factors and bacterial taxa was controlled for age. A small number 466 of bacterial taxa were significantly associated with WAZ $(N=1), \operatorname{HAZ}(N=7)$, and WHZ $(N=3)$

467 (Supplementary Table 3, columns WAZ.p, HAZ.p, WHZ.p, yellow box) but none of the associations remained significant after correction for multiple testing by the FDR method (Supplementary Table 3, columns WAZ.p.fdr, HAZ.p.fdr, and WHZ.p.fdr). We also tested for an association between microbial diversity indexes and our three multiple explanatory variables for WAZ, HAZ, and WHZ, controlled for age (Supplementary Figure 5). For this 
472 analysis the same alpha diversity indexes were used as shown in Figure 1. Fisher's Alpha

473 (Supp Fig 5.a), Richness index (Supp Fig 5.c), and Chao1 (Supp Fig 5.d) were all highly

474 significantly associated with age. However, none of the alpha community diversities (i.e.,

475 Fisher's alpha, Simpson's index, Richness, and Chao1) remained significantly associated with

476 WAZ, HAZ, and WHZ after controlling for age (Supplementary Figures 5.a to 5.d).

477

478 
Supplmentary Table 3. Multivariate linear regression (MRL) did not identify an association between bacterial taxa and WAZ, HAZ, and WHZ

\begin{tabular}{|c|c|c|c|c|c|c|c|c|c|c|c|c|c|}
\hline Taxa & age.p & WAZ.p & HAZ.p & WHZ.p & (Intercept).c & age.c & WAZ.c & HAZ.c & WHZ.c & age.p.fdr & WAZ.p.fdr & HAZ.p.fdr & WHZ.p.fdr \\
\hline Prevotella_copri_35.153 & 0 & 0.356 & 0.56 & 0.701 & 25.75 & 0.361 & -0.851 & -0.388 & -0.283 & 0 & 0.94184 & 0.97604 & 0.96837 \\
\hline Faecalibacterium_prausnitzii_11.383 & 0 & 0.763 & 0.835 & 0.155 & 5.397 & 0.266 & 0.112 & 0.056 & -0.425 & 0 & 0.94184 & 0.97604 & 0.68333 \\
\hline Prevotella_stercorea_7.054 & 0 & 0.775 & 0.509 & 0.879 & 3.061 & 0.182 & 0.109 & -0.181 & -0.046 & 0 & 0.94184 & 0.97604 & 0.96837 \\
\hline Bacteroides_4.636 & 0 & 0.15 & 0.802 & 0.66 & 9.53 & -0.198 & 0.661 & 0.083 & -0.162 & 0 & 0.94184 & 0.97604 & 0.96837 \\
\hline Bifidobacterium_4.117 & 0 & 0.823 & 0.23 & 0.144 & 16.033 & -0.537 & 0.084 & -0.325 & 0.441 & 0 & 0.94184 & 0.71176 & 0.68333 \\
\hline Escherichia_coli_3.505 & 0 & 0.488 & 0.098 & 0.423 & 8.359 & -0.206 & -0.261 & 0.45 & 0.242 & 0 & 0.94184 & 0.49 & 0.81346 \\
\hline Succinivibrio_dextrinosolvens_2.424 & 0 & 0.905 & 0.925 & 0.802 & -0.48 & 0.131 & 0.034 & -0.019 & -0.057 & 0 & 0.94184 & 0.97604 & 0.96837 \\
\hline Paraprevotella_xylaniphila_2.02 & 0 & 0.376 & 0.655 & 0.946 & -0.446 & 0.11 & -0.159 & 0.058 & 0.01 & 0 & 0.94184 & 0.97604 & 0.96837 \\
\hline Sutterella_wadsworthensis_1.751 & 0 & 0.793 & 0.469 & 0.122 & 3.486 & -0.078 & -0.035 & -0.069 & 0.164 & 0 & 0.94184 & 0.97604 & 0.67778 \\
\hline Streptococcus_salivarius_1.137 & 0 & 0.322 & 0.037 & 0.823 & 1.947 & -0.041 & 0.121 & -0.185 & -0.022 & 0 & 0.94184 & 0.28571 & 0.96837 \\
\hline Megasphaera_elsdenii_0.9 & 0 & 0.488 & 0.859 & 0.453 & 3.726 & -0.122 & 0.091 & 0.017 & 0.079 & 0 & 0.94184 & 0.97604 & 0.82679 \\
\hline Ruminococcaceae_UCG002_0.716 & 0 & 0.923 & 0.591 & 0.463 & -0.366 & 0.052 & -0.008 & 0.03 & 0.046 & 0 & 0.94184 & 0.97604 & 0.82679 \\
\hline Thalassospira_0.707 & 0 & 0.419 & 0.886 & 0.184 & -0.087 & 0.037 & 0.067 & 0.009 & -0.088 & 0 & 0.94184 & 0.97604 & 0.68333 \\
\hline Eubacterium_rectale_0.673 & 0 & 0.373 & 0.162 & 0.055 & -0.165 & 0.042 & -0.067 & 0.076 & 0.116 & 0 & 0.94184 & 0.675 & 0.475 \\
\hline Prevotella_ruminicola_0.615 & 0 & 0.302 & 0.451 & 0.921 & 0.25 & 0.021 & 0.065 & 0.034 & 0.005 & 0 & 0.94184 & 0.97604 & 0.96837 \\
\hline Pseudobutyrivibrio_ruminis_0.568 & 0 & 0.892 & 0.028 & 0.292 & -0.252 & 0.043 & -0.008 & 0.095 & 0.051 & 0 & 0.94184 & 0.28571 & 0.68333 \\
\hline Lactobacillus_ruminis_0.544 & 0 & 0.865 & 0.264 & 0.24 & 1.877 & -0.055 & -0.019 & 0.088 & 0.103 & 0 & 0.94184 & 0.73333 & 0.68333 \\
\hline Roseburia_inulinivorans_0.465 & 0 & 0.49 & 0.91 & 0.882 & -0.058 & 0.023 & -0.033 & -0.004 & 0.006 & 0 & 0.94184 & 0.97604 & 0.96837 \\
\hline Holdemanella_biformis_0.416 & 0 & 0.732 & 0.585 & 0.512 & -0.067 & 0.023 & 0.015 & -0.018 & 0.024 & 0 & 0.94184 & 0.97604 & 0.85333 \\
\hline Allisonella_histaminiformans_0.421 & 0 & 0.093 & 0.001 & 0.949 & 0.895 & -0.023 & 0.07 & -0.1 & 0.002 & 0 & 0.94184 & 0.05 & 0.96837 \\
\hline Collinsella_aerofaciens_0.396 & 0 & 0.989 & 0.458 & 0.705 & 1.194 & -0.037 & 0.001 & -0.023 & 0.013 & 0 & 0.989 & 0.97604 & 0.96837 \\
\hline Erysipelotrichaceae_sp_0.318 & 0 & 0.421 & 0.071 & 0.51 & -0.014 & 0.015 & -0.028 & 0.046 & -0.019 & 0 & 0.94184 & 0.44375 & 0.85333 \\
\hline Veillonella_dispar_0.314 & 0 & 0.67 & 0.209 & 0.276 & 0.578 & -0.015 & 0.017 & -0.036 & -0.035 & 0 & 0.94184 & 0.69667 & 0.68333 \\
\hline Megamonas_funiformis_0.308 & 0 & 0.445 & 0.022 & 0.988 & 0.937 & -0.031 & 0.054 & -0.118 & 0.001 & 0 & 0.94184 & 0.28571 & 0.988 \\
\hline Phascolarctobacterium_succinatutens_0.284 & 0 & 0.537 & 0.717 & 0.896 & -0.032 & 0.016 & 0.043 & -0.018 & 0.007 & 0 & 0.94184 & 0.97604 & 0.96837 \\
\hline Veillonella_ratti_0.302 & 0 & 0.569 & 0.685 & 0.265 & 1.476 & -0.052 & -0.045 & 0.023 & 0.07 & 0 & 0.94184 & 0.97604 & 0.68333 \\
\hline Lachnospiraceae_sp_0.295 & 0 & 0.817 & 0.636 & 0.02 & 0.45 & -0.008 & 0.004 & -0.005 & -0.029 & 0 & 0.94184 & 0.97604 & 0.33333 \\
\hline Eubacterium_coprostanoligenes_0.278 & 0 & 0.225 & 0.843 & 0.281 & -0.232 & 0.024 & 0.03 & 0.003 & -0.021 & 0 & 0.94184 & 0.97604 & 0.68333 \\
\hline Pasteurella_multocida_0.249 & 0 & 0.86 & 0.983 & 0.71 & -0.275 & 0.024 & 0.012 & 0.001 & -0.021 & 0 & 0.94184 & 0.983 & 0.96837 \\
\hline Ruminococcaceae_sp_0.231 & 0 & 0.539 & 0.977 & 0.837 & 0.004 & 0.011 & 0.016 & 0.001 & -0.004 & 0 & 0.94184 & 0.983 & 0.96837 \\
\hline Olsenella_sp.SK9K4_0.238 & 0 & 0.78 & 0.87 & 0.328 & 1.019 & -0.034 & 0.016 & -0.007 & 0.045 & 0 & 0.94184 & 0.97604 & 0.68333 \\
\hline Megasphaera_micronuciformis_0.233 & 0 & 0.28 & 0.03 & 0.003 & 1.098 & -0.034 & 0.076 & -0.11 & 0.165 & 0 & 0.94184 & 0.28571 & 0.125 \\
\hline Lachnoclostridium_sp_0.215 & 0 & 0.108 & 0.04 & 0.605 & 0.069 & 0.007 & -0.019 & 0.018 & 0.005 & 0 & 0.94184 & 0.28571 & 0.96837 \\
\hline Parabacteroides_merdae_0.215 & 0 & 0.593 & 0.242 & 0.323 & 0.664 & -0.019 & 0.028 & -0.044 & 0.041 & 0 & 0.94184 & 0.71176 & 0.68333 \\
\hline Oscillibacter_sp_0.202 & 0 & 0.735 & 0.576 & 0.634 & -0.012 & 0.01 & -0.008 & 0.01 & 0.009 & 0 & 0.94184 & 0.97604 & 0.96837 \\
\hline Prevotella_nanceiensis_0.285 & 0.005 & 0.359 & 0.198 & 0.725 & -0.048 & 0.015 & 0.073 & -0.074 & -0.022 & 0.0069444 & 0.94184 & 0.69667 & 0.96837 \\
\hline Streptococcus_equinus_0.895 & 0.015 & 0.874 & 0.763 & 0.743 & 1.43 & -0.029 & -0.028 & -0.039 & -0.047 & 0.02027 & 0.94184 & 0.97604 & 0.96837 \\
\hline Ruminococcus_torques_0.411 & 0.038 & 0.158 & 0.852 & 0.057 & 0.308 & 0.005 & 0.05 & -0.005 & -0.054 & 0.05 & 0.94184 & 0.97604 & 0.475 \\
\hline Ruminococcus_gnavus_0.311 & 0.046 & 0.646 & 0.639 & 0.209 & 0.421 & -0.007 & -0.025 & 0.019 & -0.056 & 0.058974 & 0.94184 & 0.97604 & 0.68333 \\
\hline Sutterella_sp.YIT_0.82 & 0.052 & 0.765 & 0.346 & 0.844 & 0.691 & 0.009 & 0.021 & 0.047 & 0.011 & 0.063415 & 0.94184 & 0.9025 & 0.96837 \\
\hline Dorea_sp_0.214 & 0.051 & 0.134 & 0.111 & 0.398 & 0.178 & 0.002 & 0.018 & -0.014 & -0.008 & 0.063415 & 0.94184 & 0.50455 & 0.796 \\
\hline Catenibacterium_mitsuokai_0.294 & 0.081 & 0.699 & 0.779 & 0.086 & 0.102 & 0.007 & 0.022 & -0.012 & -0.079 & 0.096429 & 0.94184 & 0.97604 & 0.5625 \\
\hline Parabacteroides_distasonis_0.474 & 0.125 & 0.336 & 0.207 & 0.09 & 0.647 & -0.005 & -0.052 & 0.049 & 0.073 & 0.14535 & 0.94184 & 0.69667 & 0.5625 \\
\hline Roseburia_faecis_0.213 & 0.229 & 0.085 & 0.512 & 0.005 & 0.129 & 0.003 & 0.067 & -0.018 & -0.088 & 0.26023 & 0.94184 & 0.97604 & 0.125 \\
\hline Fusobacterium_mortiferum_0.643 & 0.263 & 0.04 & 0.092 & 0.298 & 0.869 & -0.013 & -0.347 & 0.205 & 0.141 & 0.29222 & 0.94184 & 0.49 & 0.68333 \\
\hline Anaerostipes_sp.5_0.27 & 0.375 & 0.573 & 0.937 & 0.245 & 0.203 & 0.001 & -0.014 & -0.001 & -0.023 & 0.40761 & 0.94184 & 0.97604 & 0.68333 \\
\hline Klebsiella_pneumoniae_0.691 & 0.41 & 0.902 & 0.022 & 0.056 & 0.87 & -0.007 & -0.017 & 0.223 & -0.207 & 0.43617 & 0.94184 & 0.28571 & 0.475 \\
\hline Clostridium_celatum_0.207 & 0.471 & 0.899 & 0.361 & 0.201 & 0.162 & 0.002 & 0.005 & 0.025 & -0.039 & 0.49062 & 0.94184 & 0.9025 & 0.68333 \\
\hline Blautia_sp_0.352 & 0.593 & 0.887 & 0.897 & 0.797 & 0.377 & -0.002 & -0.009 & -0.006 & -0.013 & 0.6051 & 0.94184 & 0.97604 & 0.96837 \\
\hline Haemophilus_parainflu & 0.706 & 0.866 & 0.857 & 0.325 & 0.862 & -0.003 & -0.018 & 0.014 & -0.083 & 0.706 & 0.94184 & 0.97604 & 0.68333 \\
\hline
\end{tabular}


483 In this study we utilised a very large cohort of children from an iron intervention trial in a

484 rural region of The Gambia. This large study gave us an excellent opportunity to analyse in

485 detail the microbiome in children in the first 40 months of life, a critical time frame in the

486 development of the gut microbiome of humans both in industrialised and non-industrialised

487 societies ${ }^{1}$. Our analyses showed that age was the main factor that determined the overall

488 bacterial composition in these samples and highlighted the particular importance of the

489 Prevotella genus. It has previously been established that the Prevotella genus can be used as

490 the most important discriminatory taxon to differentiate between rural Africans and people

491 from industrialised countries and that this separation defined by Prevotella is not just limited

492 to Africa ${ }^{1,2,22}$. This study however shows how these Prevotella-rich gut microbiome

493 compositions develop over time; it shows that Prevotella copri rises to dominance rapidly in

494 the first 12 months and remains the most abundant species during the remainder of early

495 childhood ( $\sim 35 \%$ of all reads) and in addition shows the steady development of a trophic

496 network of species centered around Prevotella stercorea in addition to the trophic network

497 centered around Faecalibacterium prausnitzii. At the age of 30 months and onwards these

498 two trophic networks together account for $\sim 38 \%$ of all reads $(20 \%+18 \%$ approximately,

499 respectively).

501 F. prausnitzii is well known to be of importance in industrialised settings and is often associated with health; it is typically absent before the age of 6 months but then appears and

503 its numbers steadily increase over time once weaning commences ${ }^{15,23}$. The F. prausnitzii

504 trophic network development seen in this study mirrors the F. prausnitzii trophic network

505 development seen in a study with Finnish and Estonian children, though perhaps reached a 
stable level about 1 year earlier. The reasons for this are that the F. prausnitzii trophic

507 network in Finnish/Estonian children can be said to be slightly more complex and as a whole

508 more abundant in terms of the percentage of reads, and that the F. prausnitzii trophic

509 network in children from The Gambia appears to utilize the metabolic activity of $P$. copri, a

510 species which independently reaches a very high stable prevalence already at the age of one

511 year. Prevotella copri, the most dominant species in this dataset, is expected to be negatively

512 correlated with most bacterial groups, even in the absence of clear antagonism, when

513 normalizing the abundances of species to $100 \%$ of all reads, due to its high abundance

514 ( 35\%) yet appears to have a somewhat positive association with the F. prausnitzii trophic

515 network (Figures 6.a and 6.f) and an non-antagonistic yet numerically expected negative

516 association with the Prevotella stercorea trophic network when looking at the bulk of the

517 samples (Figures 6.b and 6.f).

518

519 The trophic network centred around Prevotella stercorea is typically not found in gut

520 microbiomes from individuals in industrialized countries. Many of the species associated in

521 this trophic network are not found in microbiota in industrialized countries, or in only low

522 numbers ${ }^{1,2,15,24}$. As a result, they have often not been studied sufficiently (or at all) which

523 makes it difficult to understand or explain, with any degree of certainty, what their roles are

524 within this trophic network. The $P$. stercorea trophic network, as described here in the

525 Gambian context, appears more diverse and self-contained than the F. prausnitzii network as

526 it does not depend on P. copri and is typically strongly negatively correlated with all other

527 main bacterial groups, apart from P. copri and the F. prausnitzii trophic network (Figure 5.b).

528 Whilst not all species within this network have been studied in detail, it is clear, from what is 
529 known about many of these species, that the assemblage should have a very wide

530 assortment of metabolic capabilities covering everything needed to form a self-contained

531 trophic network, leading (for example) to an enhanced production of short chain fatty acids,

532 as compared to microbiome compositions lacking this trophic network.

533 A particularly strong and important factor in the composition of the gut microbiome in these

534 children from The Gambia is the clear antagonistic relationship between the Prevotella and

535 Bacteroides genera (Figures 5 and 6). The Prevotella copri and the Prevotella stercorea

536 network are both very strongly negatively correlated with the abundance of Bacteroides

537 (Spearman rho $=-0.36$ and -0.32 , respectively). The F. prausnitzii network however is not

538 negatively correlated with Bacteroides, similar to the association commonly found between

539 F. prausnitzii and Bacteroides in gut microbiome compositions from industrialized countries

540 where it is often found to be positive.

541

542 Similar to infant microbiotas in industrialized countries, the Bifidobacterium genus is the

543 most important and dominant group early in infancy. As infants are weaned, the dominance

544 of Bifidobacteria wanes and they are rapidly replaced by bacteria associated with a more

545 adult-like "African" microbiome composition, especially in the case of these Gambian

546 children ${ }^{2}$. Bifidobacterium levels at 3 years of age are much lower in children from The

547 Gambia than in European children, possibly because the lactate- and acetate-producing

548 Bifidobacteria become part of the trophic networks found in Europe whereas they are

549 replaced by other species with a similar function (acetate and lactate production) in The

550 Gambia. The higher abundance of Bifidobacterium in European children may also be a result

551 of earlier and longer formula-feeding in those children ${ }^{25,26}$. 
552 A decrease was also seen in the abundance of several potentially pathogenic species (e.g., $E$.

553 coli and Sutterella), the Bacteroides genus, and several other infant/baby-associated

554 microorganisms.

555

556 The high abundance of $P$. copri in the The Gambia cohort emphasises the function of $P$. copri

557 and their metabolomic pathways in fermenting complex polysaccharides, which is important

558 for people with a high fibre diet. It has also been shown in other studies that Prevotella

559 species play an important role in glucose metabolism improvements after the consumption

560 of barley kernel-based bread ${ }^{27}$. Metatranscriptomic RNA sequencing has provided detailed

561 information showing that $P$. copri encodes all the enzymes necessary for the Embden-

562 Meyerhof-Parnas (EMP) pathway which is the most recognised glycolytic pathway ${ }^{28}$ and is

563 based on glycolysis and succinate production from fumarate. In addition, transcriptomic

564 profiling has further revealed that it can degrade pyruvate to acetate and formate ${ }^{29}$. Another

565 study looking at sports athletes associated Prevotella with a number of amino acid and

566 carbohydrate metabolism pathways including lysine biosynthesis, alanine, aspartate and

567 glutamate metabolism, and D-glutamine and D-glutamate metabolism. Prevotella is also

568 known to be important in multiple pathways involved in drug metabolism, carbohydrate

569 metabolism, and metabolism of cofactors and vitamins, including vitamin B6 metabolism ${ }^{30}$.

570

571 In conclusion, we have analysed a stool collection from children between the age of 7 and 40

572 months of the upper region of The Gambia with a high fiber diet; the largest African

573 paediatric microbiome collection to date. Detailed analysis of the gut microbiome has

574 provided insights into the development of a Prevotella-rich gut microbiome. We observed

575 similar changes to those described in paediatric cohorts from industrialised countries, such as 
576 the shift from Bifidobacteria to the development of trophic network centered around $F$.

577 prausnitzii. However, in addition we were able to describe the steady development of a

578 trophic network centered around $P$. stercorea and the rapid rise to dominance of $P$. copri.

579 The high abundance of Prevotella in non-industrialized countries makes this genus the most

580 discriminating taxon between a healthy high-fiber diet and an unhealthy low-fiber diet seen

581 in industrialized countries. In industrialized countries, the increase of various diseases of

582 affluence, like diabetes, is typically associated with the (partial) loss of the last trophic

583 network (indicated by lower F. prausnitzii numbers) and an increase of Bacteroides and/or

584 Proteobacteria. The parallel loss of $P$. copri and the $P$. stercorea trophic network, whose

585 development is described here, indicates the likely importance of the metabolic pathways

586 and capabilities of Prevotella and species associated with them, both for maintaining a higher

587 fermentative capacity (SCFA production), a healthier gut microbiome environment and for

588 keeping opportunistic pathogens at bay.

589

590

591 
593 We acknowledge the staff of the MRC Unit The Gambia at LSHTM and, in particular, the

594 committed IHAT-GUT field team, for their support of, and their invaluable contribution to,

595 this research. We thank the staff of the Yorrobawol health centre, Darsilami community

596 health post, Konkuba community health post, Taibatu health post and Chamoi Health Centre

597 for welcoming our team and supporting our study. We thank the local communities and the

598 many study participants and their families in the Wuli and Sandu districts of the URR, The

599 Gambia, who have been so willing to contribute to this study.

600 This study is registered at Clinical Trials. gov as NCT02941081.

\section{Competing interests}

602 D.I.A.P. is one of the inventors of the IHAT iron supplementation technology for which she 603 could receive future awards to inventors through the MRC Awards to Inventor scheme.

604 D.I.A.P. has served as a consultant for Vifor Pharma UK, Shield Therapeutics, Entia Ltd, 605 Danone Nutritia, UN Food and Agriculture Organization (FAO) and Nemysis Ltd. D.I.A.P. has

606 since moved to full employment with Vifor Pharma UK, but all work pertaining to this study

607 was conducted independently of Vifor Pharma.

608 Notwithstanding, the authors declare no potential conflicts of interest with respect to the

609 research, authorship, and/or publication of this article.

610

\section{Contributors}

612 Josef Wagner, data analysis and original draft preparation

613 Marcus De Goffau, data analysis and manuscript review \& editing

614 Julian Parkhill, involved in initial study set up and critical review of the manuscript 
615 Dora I.A. Pereira, conceptualization, data curation, funding acquisition, methodology, project

616 administration, supervision, visualization, writing - review \& editing

617 Chilel Sanyang, investigation, methodology, writing - review \& editing

618 Amadou T. Jallow, investigation, methodology, supervision, validation, writing - review \&

619 editing

620 Andrew M. Prentice, conceptualization, funding acquisition, supervision, writing - review \&

621 editing

622

623

624

625 Funding

626 This IHAT-GUT study was supported by a Bill \& Melinda Gates Foundation Grand Challenges

627 New Interventions in Global Health award [OPP1140952].

628 The Nutrition Group of the MRC Unit The Gambia at LSHTM are supported by core funding

629 MC-A760-5QX00 to the MRC Unit The Gambia/MRC International Nutrition Group by the UK

630 MRC and the UK Department for the International Development (DFID) under the MRC/DFID

631 Concordat agreement. The funders had no role in study design, data collection and analysis,

632 decision to publish, or preparation of the manuscript.

633

634 Ethics approval

635 The study protocol and any subsequent amendments have been reviewed and approved by

636 The Gambia Government/MRC Joint Ethics Committee (reference SCC1489). Clinical Trials

637 Authorization has been granted by the Medicines Control Agency, The Gambia

$638(\mathrm{HP373} / 347 / 16 / \mathrm{MJK}(80))$.

639 
641 1. Yatsunenko, T. et al. Human gut microbiome viewed across age and geography. Nature 486, 222-227 (2012).

643 2. De Filippo, C. et al. Impact of diet in shaping gut microbiota revealed by a comparative study in children from Europe and rural Africa. Proc. Natl. Acad. Sci. 107, 14691-14696

645 (2010).

646

3. Ursell, L. K., Metcalf, J. L., Parfrey, L. W. \& Knight, R. Defining the human microbiome.

647 Nutr. Rev. (2012). doi:10.1111/j.1753-4887.2012.00493.x

648

4. Siezen, R. J. \& Kleerebezem, M. The human gut microbiome: are we our enterotypes? Microb. Biotechnol. 4, 550-553 (2011).

650

5. Arumugam, M. et al. Enterotypes of the human gut microbiome. Nature 473, $174-180$

651 (2011).

652 6. Wu, G. D. et al. with Gut Microbial Enterotypes. Science (80-. ). (2011).

653 7. Gorvitovskaia, A., Holmes, S. P. \& Huse, S. M. Interpreting Prevotella and Bacteroides 654 as biomarkers of diet and lifestyle. Microbiome 4, 15 (2016).

655

8. Costea, P. I. et al. Enterotypes in the landscape of gut microbial community 656 composition. Nat. Microbiol. (2017). doi:10.1038/s41564-017-0072-8

657

9. Koren, O. et al. A Guide to Enterotypes across the Human Body: Meta-Analysis of Microbial Community Structures in Human Microbiome Datasets. PLoS Comput. Biol. (2013). doi:10.1371/journal.pcbi.1002863

660

10. Zhou, Y. et al. Exploration of bacterial community classes in major human habitats. Genome Biol. (2014). doi:10.1186/gb-2014-15-5-r66

662 11. Knights, D. et al. Rethinking enterotypes. Cell Host and Microbe (2014). 
664 12. Tyakht, A. V. et al. Human gut microbiota community structures in urban and rural

665

666

667

668

669

670

671

672

673

674

675

676

677

678

679

680

681

682

683

684

685

686

687 populations in Russia. Nat. Commun. (2013). doi:10.1038/ncomms3469

13. Tyakht, A. V, Alexeev, D. G., Popenko, A. S., Kostryukova, E. S. \& Govorun, V. M. Rural and urban microbiota. Gut Microbes (2014). doi:10.4161/gmic.28685

14. Pereira, D. I. A. et al. A novel nano-iron supplement to safely combat iron deficiency and anaemia in young children: The IHAT-GUT double-blind, randomised, placebocontrolled trial protocol. Gates Open Res. (2018). doi:10.12688/gatesopenres.12866.2

15. Ruohtula, T. et al. Maturation of Gut Microbiota and Circulating Regulatory T Cells and Development of IgE Sensitization in Early Life. Front. Immunol. 10, 2494 (2019).

16. The Gambia Bureau of Statistics (GBOS), I. I. The Gambia Demographic and Health Survey 2013. (2014).

17. Eriksen, K. G. et al. Following the World Health Organization's Recommendation of Exclusive Breastfeeding to 6 Months of Age Does Not Impact the Growth of Rural Gambian Infants. J. Nutr. 147, 248-255 (2017).

18. Prentice, A. M. \& Paul, A. A. Fat and energy needs of children in developing countries. Am. J. Clin. Nutr. 72, 1253s-1265s (2000).

19. Mandal, S. et al. Analysis of composition of microbiomes: a novel method for studying microbial composition. Microb. Ecol. Heal. Dis. 26, 27663 (2015).

20. Steffan, S. A. et al. Microbes are trophic analogs of animals. Proc. Natl. Acad. Sci. 112, 15119-15124 (2015).

21. Hui, D. Food Web: Concept and Applications. Nat. Educ. Knowl. 3, 6 (2012).

22. Schnorr, S. L. et al. Gut microbiome of the Hadza hunter-gatherers. Nat. Commun. (2014). doi:10.1038/ncomms4654

23. Miquel, S. et al. Ecology and metabolism of the beneficial intestinal commensal 
689 24. Schnorr, S. L. et al. Gut microbiome of the Hadza hunter-gatherers. Nat. Commun. 5, 3654 (2014).

691 25. Nagpal, R. et al. Evolution of gut Bifidobacterium population in healthy Japanese infants over the first three years of life: A quantitative assessment. Sci. Rep. (2017). doi:10.1038/s41598-017-10711-5

26. Gueimonde, M., Laitinen, K., Salminen, S. \& Isolauri, E. Breast milk: A source of bifidobacteria for infant gut development and maturation? Neonatology (2007). doi:10.1159/000100088

697

27. Kovatcheva-Datchary, P. et al. Dietary Fiber-Induced Improvement in Glucose Metabolism Is Associated with Increased Abundance of Prevotella. Cell Metab. 22, 971-982 (2015).

700

28. Wolfe, A. J. Glycolysis for the Microbiome Generation. in Metabolism and Bacterial Pathogenesis (ed. Cohen, T. C. P.) (ASM Press, 2015). doi:https://doi.org/10.1128/9781555818883.ch1

29. Franke, T. \& Deppenmeier, U. Physiology and central carbon metabolism of the gut bacterium Prevotella copri. Mol. Microbiol. 109, 528-540 (2018).

30. Petersen, L. M. et al. Community characteristics of the gut microbiomes of competitive cyclists. Microbiome 5, 98 (2017). 
713 Supplementary Figure 3. Eight species were statistically significantly different between the

\section{D1 and D85 timepoints}

715 Mixed Effect Regression modelling (MERM) was used to analyse samples from paired

716 repeated timepoints. Six species significantly increased in the paired sample analysis from D1

717 to Day 85 (Sup Fig 3 a - f) and two species significant decreased in the paired sample analysis

718 from D1 to Day 85 (Sup Fig 3 g-h). MERM was also used to look at the changes in alpha

719 diversity indexes in the D1/D85 paired samples set. Three alpha diversity indexes significantly

720 increased from D1 to D85 which were Richness, Chao1, and Fisher's Alpha, whereas

721 Simpson's index did not change (Sup Fig $3 \mathrm{i}-\mathbf{I})$.

722 Supplementary Figure 5. Multivariate linear regression (MLR) analysis for alpha diversity

723 indexes with age, WAZ, HAZ, and WHZ

724 MLR was performed to identify associations between bacterial taxa, community alpha

725 indexes and clinical factors for WAZ, HAZ, and WHZ. The different diversity indexes were controlled for age. Sup Fig 5.a shows the MLR model for Fisher's Alpha age + WAZ + HAZ + WHZ. Sup Fig 5.b shows the MLR model for Simpson's index age + WAZ + HAZ + WHZ. Sup

Fig 5.c shows the MLR model for Richness index age + WAZ + HAZ + WHZ, and Sup Fig 5.a shows the MLR model for Chao1 index age + WAZ + HAZ + WHZ.

730

Supplementary Table 2. Non-parametric Kruskal-Wallis rank test to identify significantly differentially abundant bacterial taxon in the 11 three-months age groups The non-parametric rank test (Kruskal-Wallis) was used to identify significantly differentially

734 abundant bacterial taxon in the 11 age groups. The first column shows all 488 species present in the entire datasets. The second column shows the relative abundance (\%). The third 
column shows the P value from the rank test. The fourth columns show the False Discovery

737 Rate (FDR) corrected $\mathrm{P}$ value. The next 11 columns show the mean abundance value in the 11 age groups (three months interval) and the last 11 columns show the median abundance value fort the same 11 age groups. The table is sorted from highest abundance species to the

740 lowest abundance species. All FDR significant species are highlighted in grey. A p value $<1 \mathrm{e}-$

74120 are shown as "0". 301/488 taxon were statistically significantly different between the 11

742 age groups. However, a large majority of them (231/301) were very low abundance taxa with an abundance below $0.1 \%$. The top 70 statistically significant species with a minimum abundance of $0.1 \%$ include $92.5 \%$ off all sequence reads.

Supplementary Table 3. Multivariate linear regression did not identify an association between bacterial taxa and WAZ, HAZ, and WHZ

MLR was used to identify an association between community composition (219/488 bacterial taxa with a minimum abundance of $0.01 \%$ ) and multiple explanatory variables for age, Weight-for-Age Z Score (WAZ), Height-for-Age Z Score (HAZ), and Weight-for-Height z-score (WHZ). Bacterial taxa were used as the dependent (response) variables and the clinical factors for age and score for WAZ, HAZ, and WHZ, were used as independent variables. The table was sorted by the false discovery rate (fdr) corrected p-values for age. The table shows the uncorrected p-values the coefficient ("c") value and the corrected p-values. MRL model was performed in Calypso Web Portal (version 8.72). The top 50 taxa are shown. Statistically significantly associated taxa with an uncorrected $p$-value $<0.05$ are highlighted in yellow and red. 

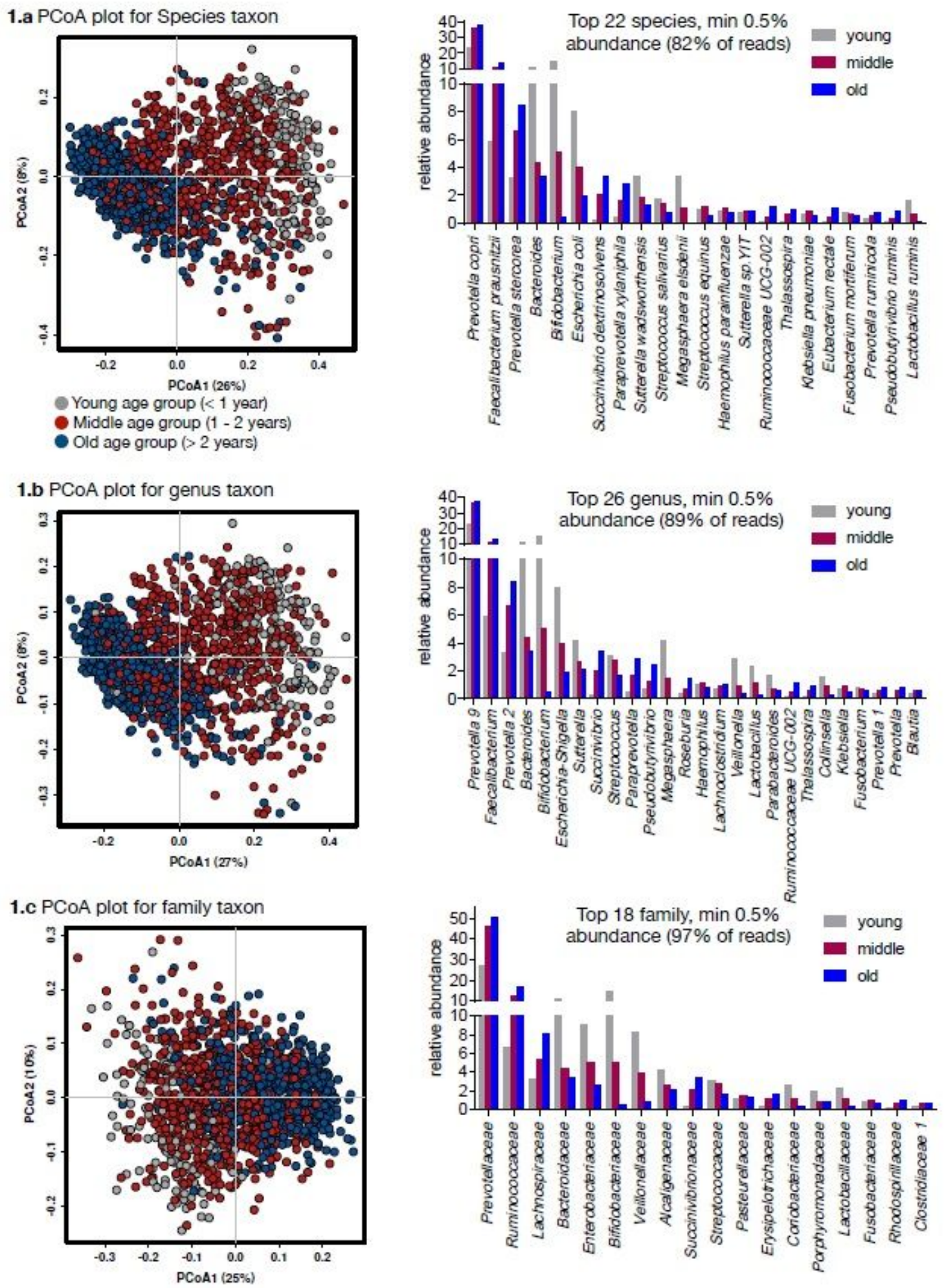

Figure 1

PCoA clustering for species, genera, and family separated into 3 age groups Principal co-ordinate analysis (PCoA) was conducted with all 1407 samples and highlights a clear separation of samples based on three age groups at the species taxonomic level (Figure 1.a), the genus taxonomic level (Figure 
1.b), and the family taxonomic level (Figure 1.c). The column graphs next to the PCoA plots show the relative abundance of the top species with a minimum abundance of $0.5 \%$ for the species taxon $(n=22)$, for the genus taxon $(n=26)$, and for the family taxon $(n=18)$. All Bacteroides species and all Bifidobacterium species were combined.
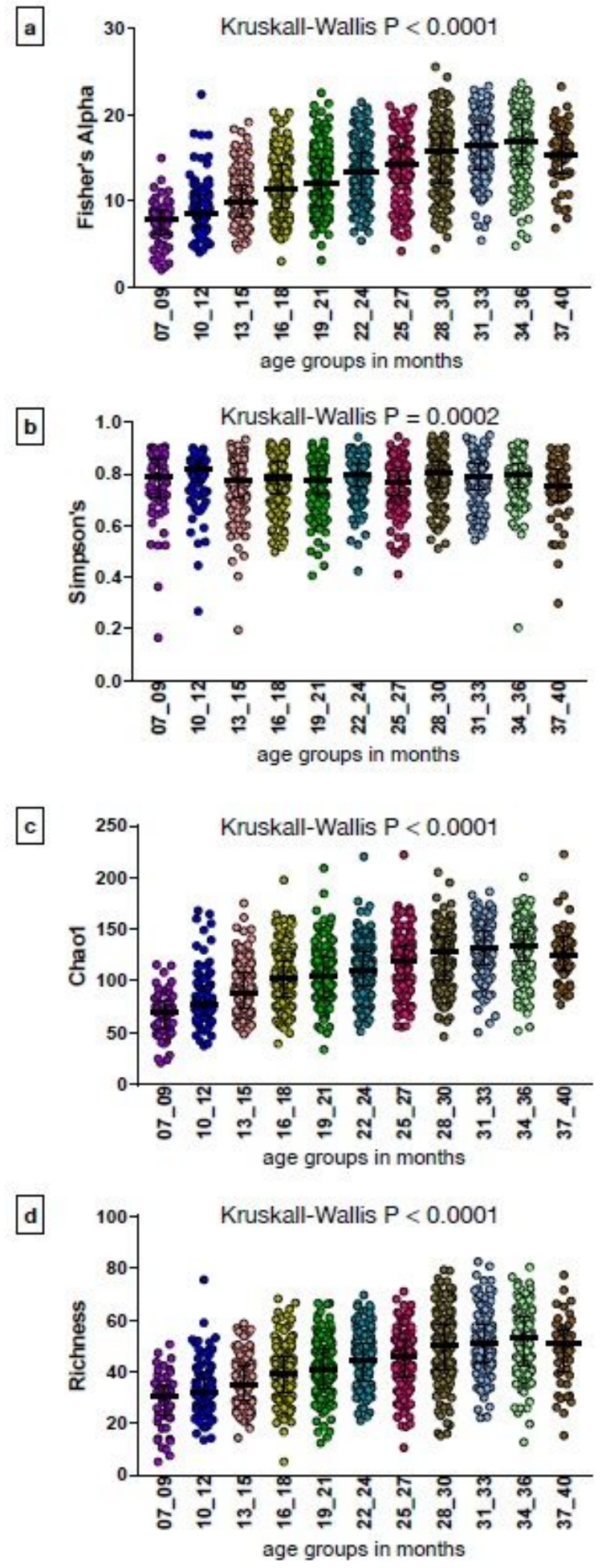

Figure 2 
Alpha diversity indexes for 11 age groups Alpha diversity was measured by two different diversity indexes and by two different richness indexes. Figure 2.a The Fisher's alpha diversity test, Figure 2.b Simpson's diversity test, Figure 2.c, Chao1 estimated richness, Figure 2.d Observed richness
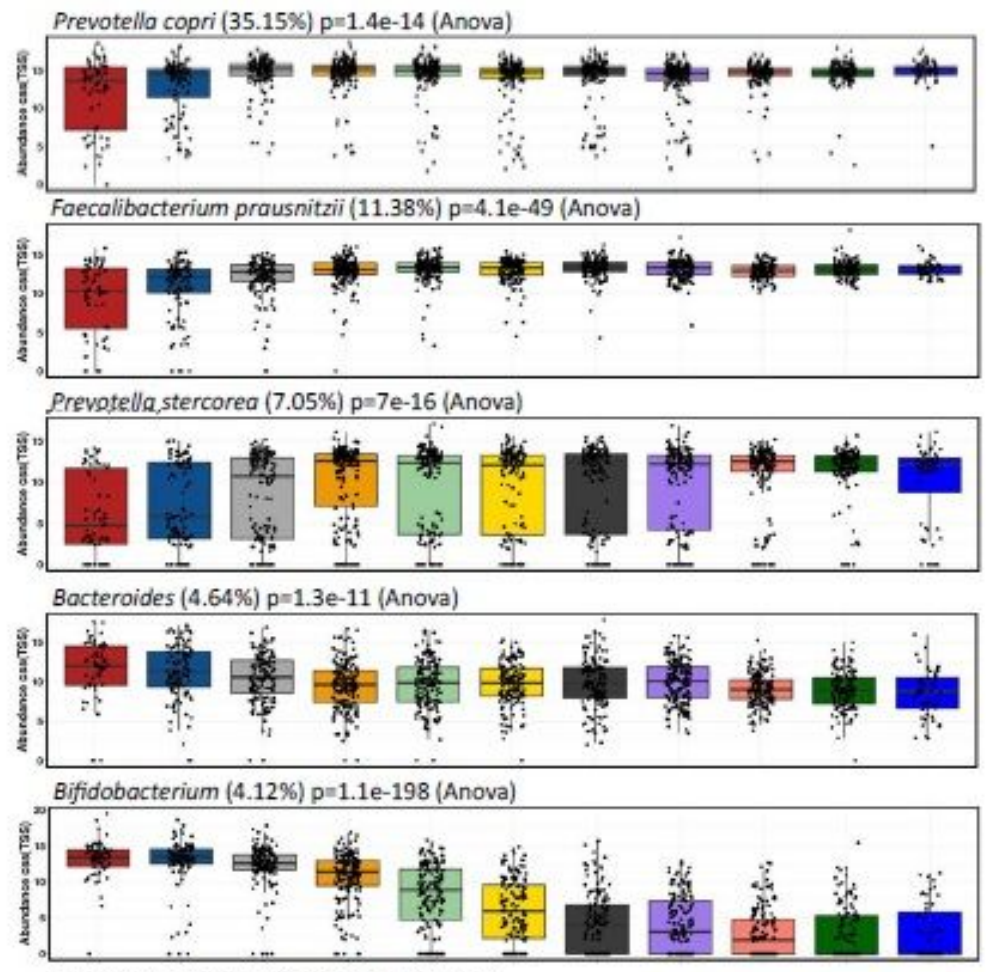

Escherichia coli (3.51\%) p=1.2e-38 (Anova)

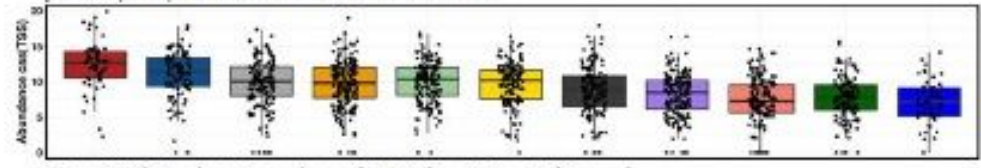

Succinivibrio dextrinosolvens (2.42\%) p=3.5e-19 (Anova)

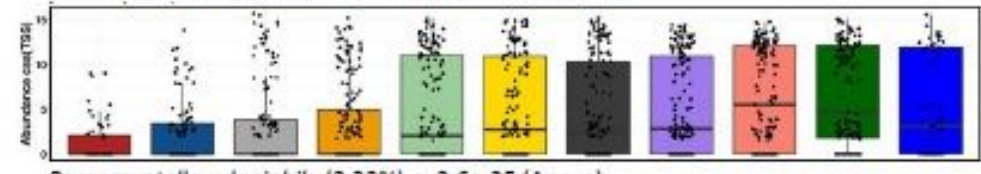

Paraprevotella xylaniphila (2.22\%) p=2.6e-35 (Anova)

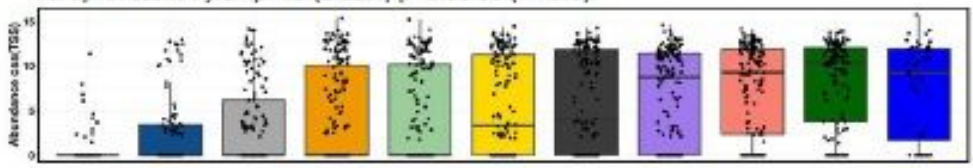

Sutterella wadsworthensis (1.75\%) p=5.2e-21 (Anova)
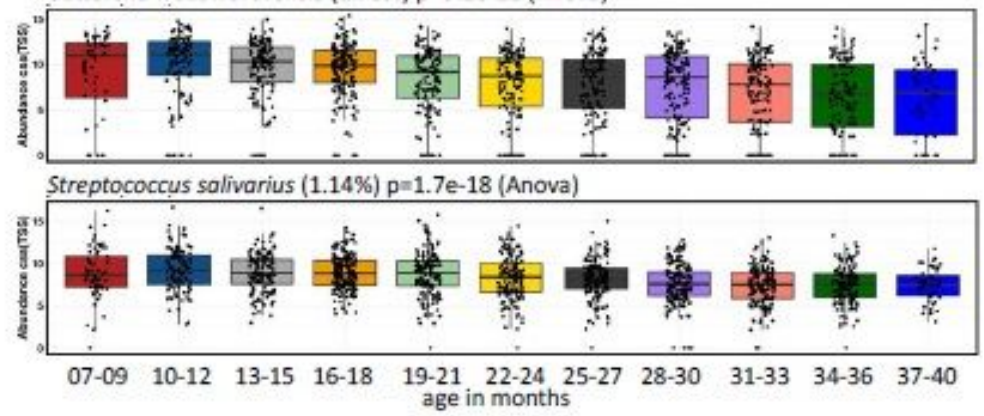

Figure 3

Top 10 species with a minimum abundance of $1 \%$, significantly associated in the 11 age groups Figure 3.a shows the significantly differentially abundant bacterial taxa using relative (total sum scaling (TSS) 
abundance. Figure 3.b shows the same dataset further transformed using cumulative sum scaling (CSS) $+\log 2$ transformation.

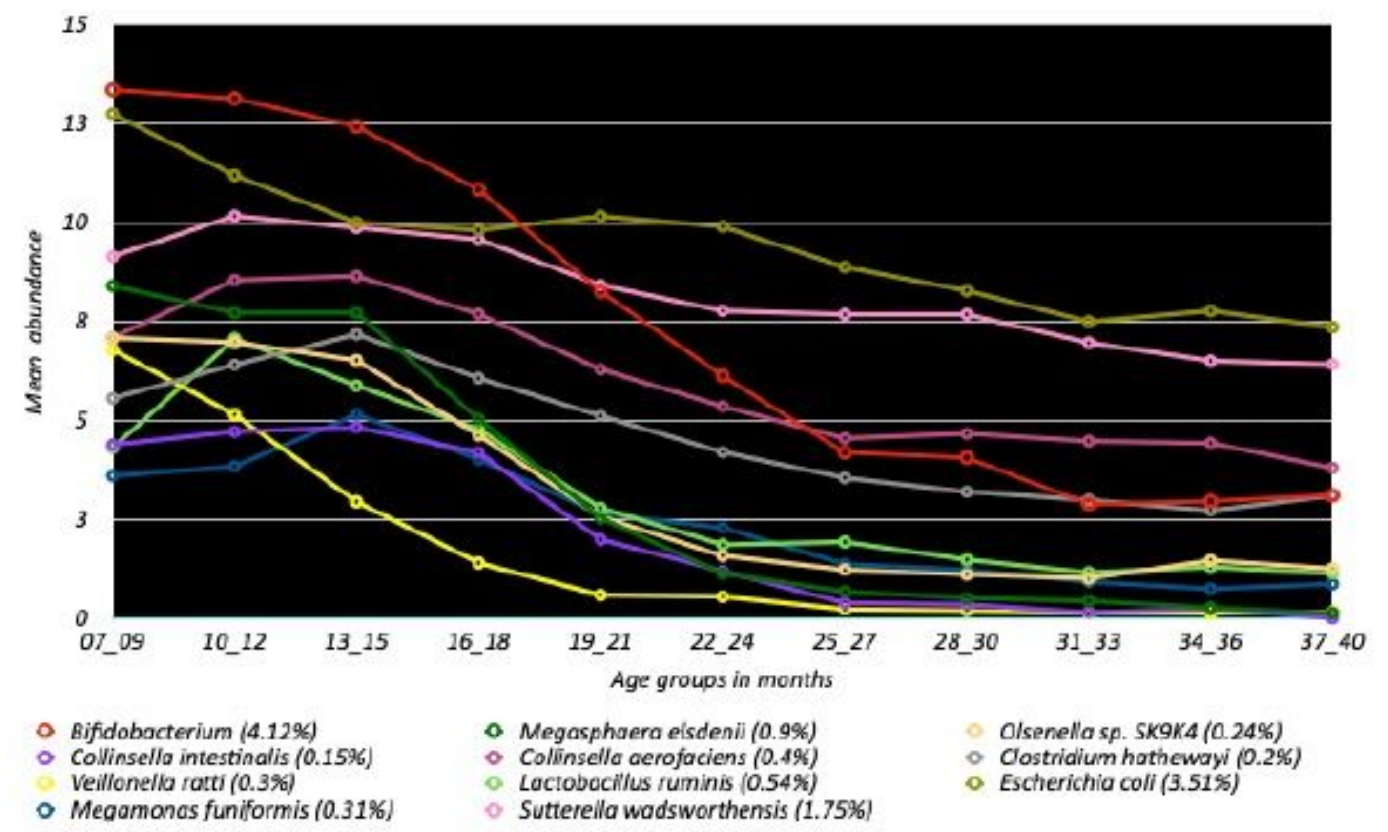

4.b Positive correlated with age with a Spearman Rho index of $\min 0.3(0.3$ to 0.56$)$ and a Spearman FDR $P<0.05$ and a minimum abundance of $0.1 \%(\mathrm{~N}=2 \mathrm{O})$

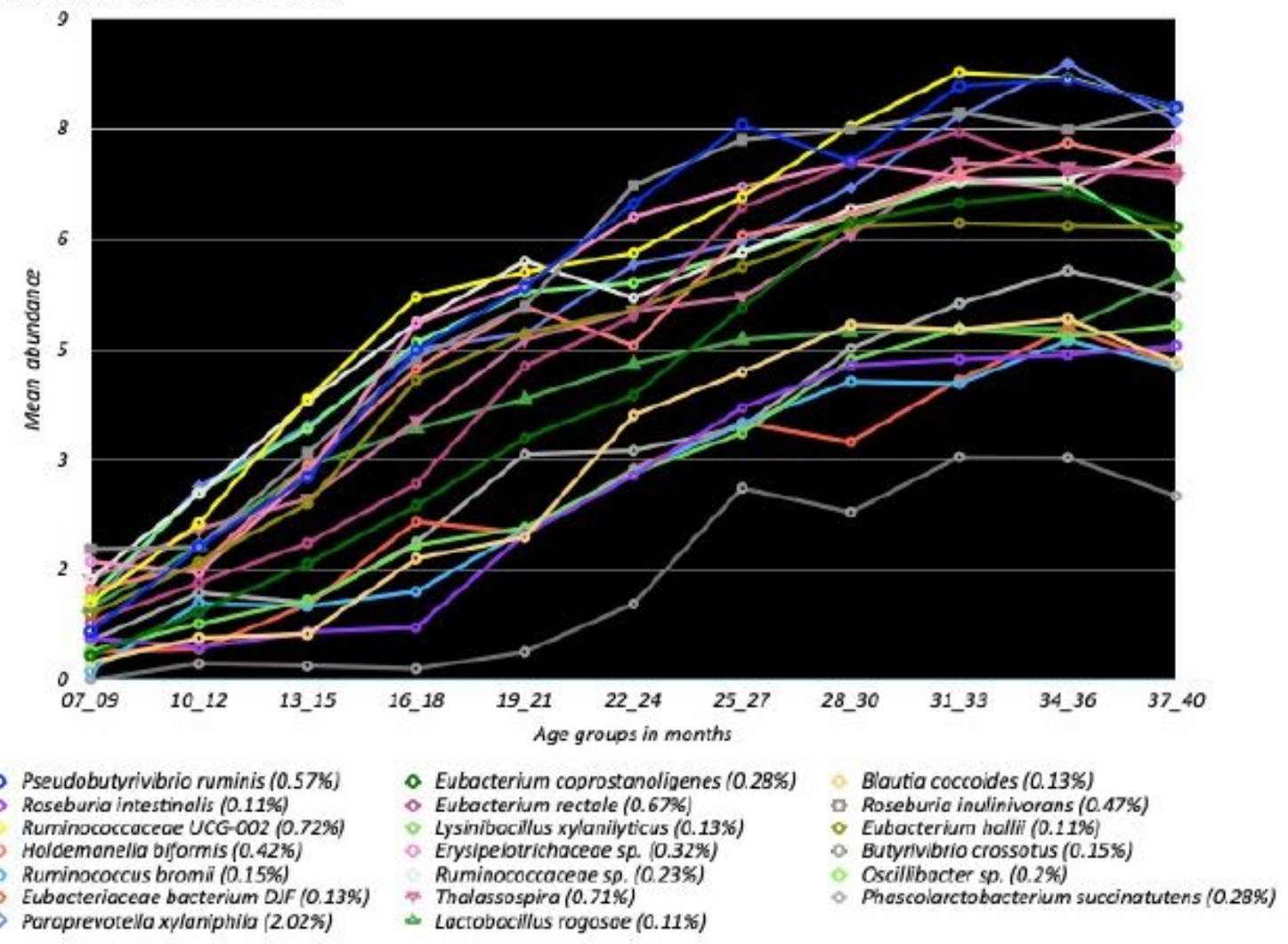

\section{Figure 4}

Top significant taxa associated with age development between the age of 7 months and 40 months. All taxa $(\mathrm{N}=11)$ associated with a decreasing abundance over time with a minimum Spearman's rho index of -0.3 from the minimum $0.1 \%$ abundance taxa dataset are presented in Figure 4.a. And, all taxa $(\mathrm{N}=20)$ 
associated with an increasing abundance over time with a minimum Spearman's rho index of 0.3 from the minimum $0.1 \%$ abundance taxa dataset are presented in Figure 4.b.
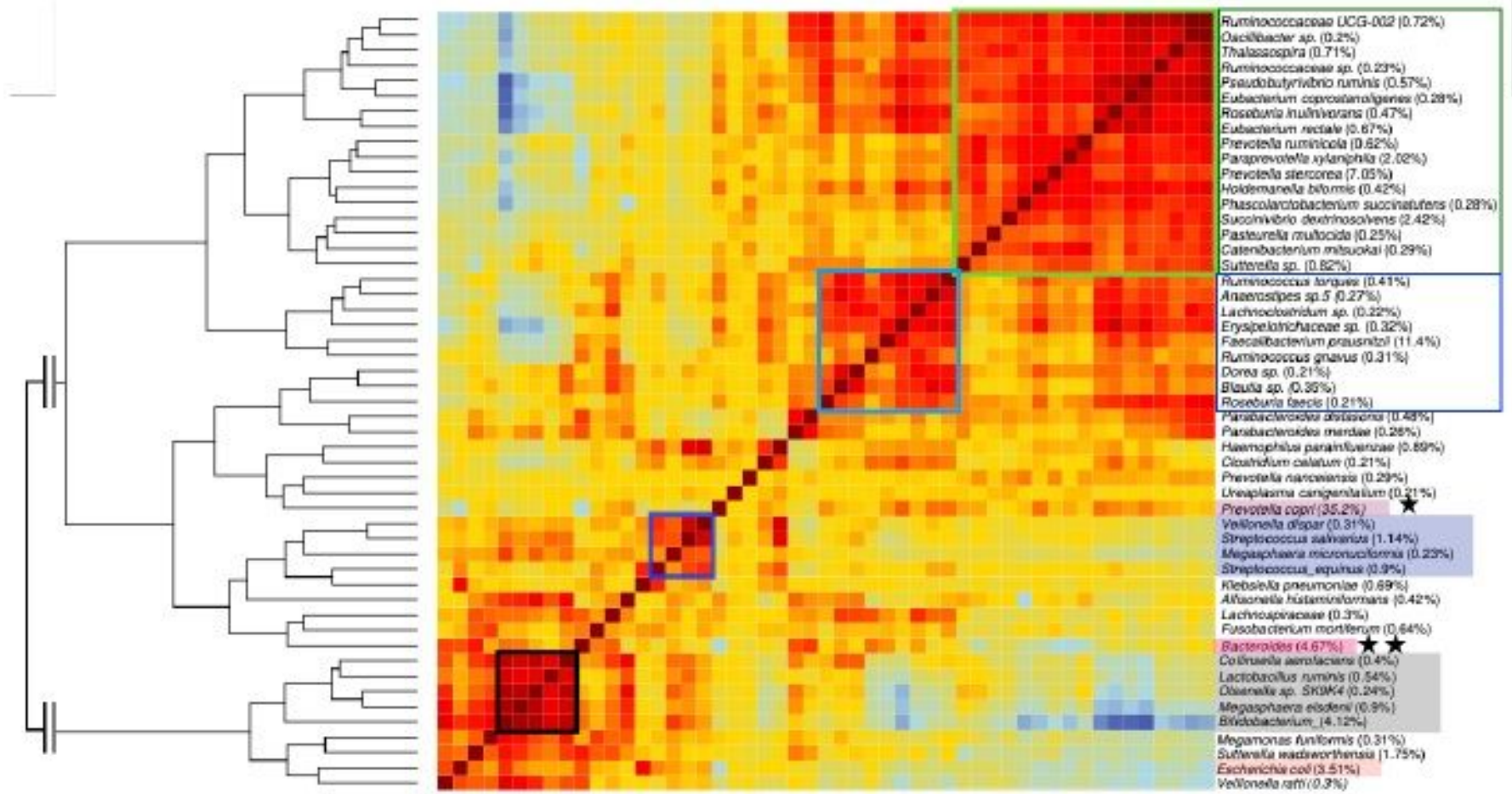

15

5.b Bacterial network - Summary

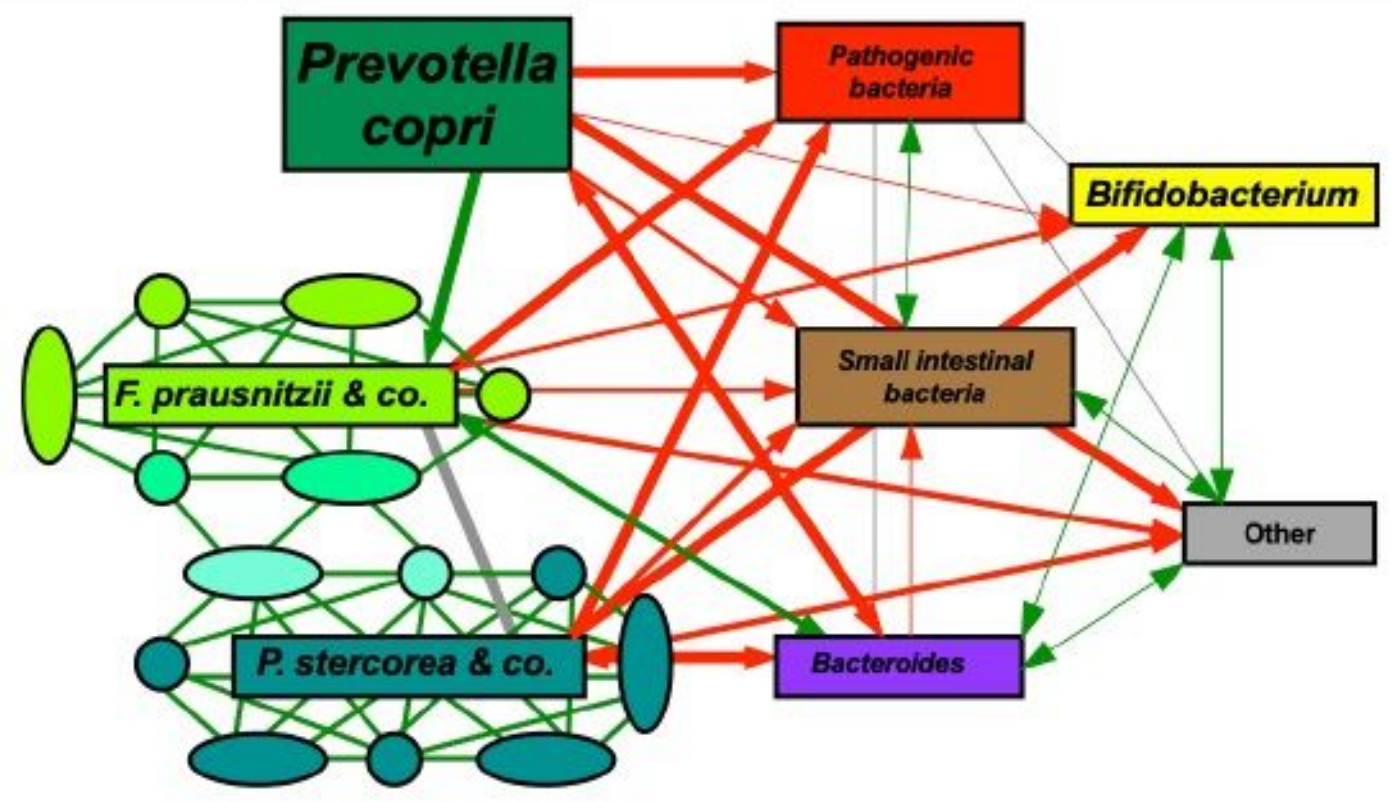

Figure 5

Species association and trophic networks Figure 5.a A network heatmap was generated using the top 51 species with a minimum abundance of $0.2 \%$ in all samples. The most dominant clusters identified in the bacterial trophic network correlation analysis are highlighted by different coloured boxes. The Prevotella stercorea network is highlighted in green, the Faecalibacterium prausnitzii network in blue, the 
Bifidobacterium network in grey, and the small intestinal microbiome network in purple. The red heatmap colour indicates strong positive correlation and the blue heatmap colour indicates strong negative correlation. There are additional species correlated outside the obvious labelled clusters, which are labelled by lines. A bacterial network summary is presented in Figure 5.b. Spearman correlation coefficient analysis was used to identify a bacterial trophic network with the strongest self-correlations. The leading species in each network is highlighted. A positive correlation is highlighted by green lines, a negative correlation is highlighted by red lines, and intermediate correlation is highlighted by grey lines
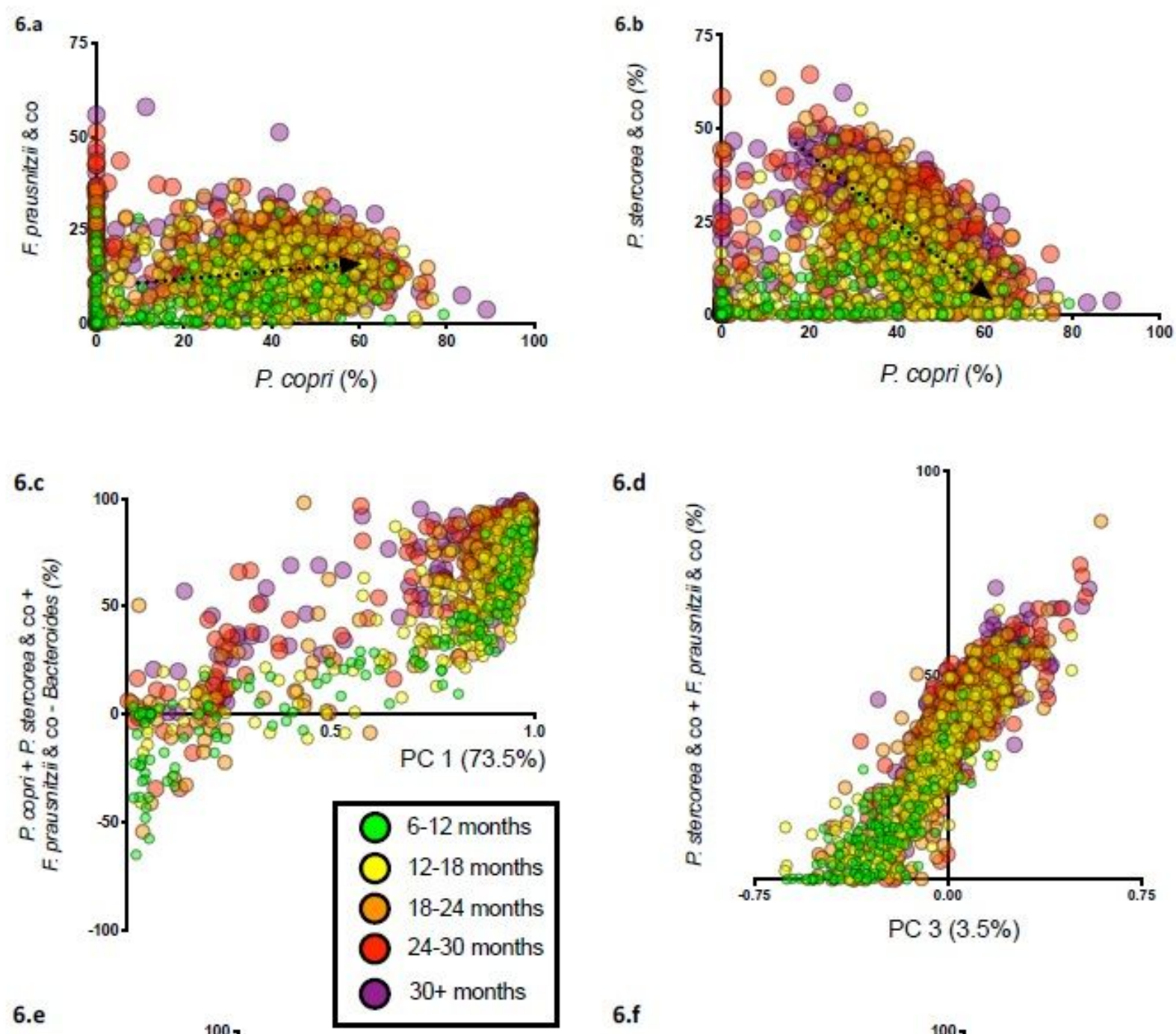

6.d
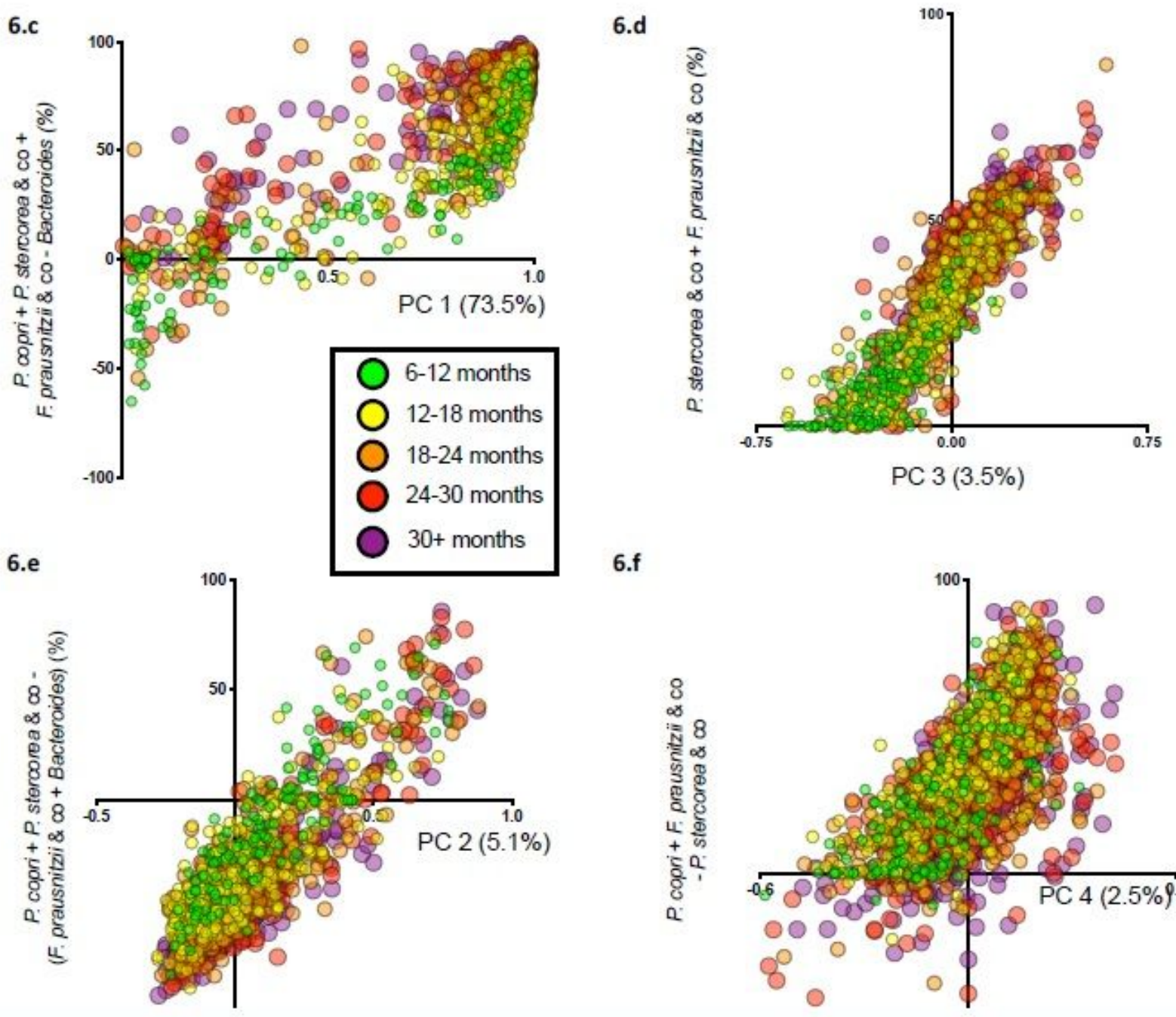

$6 . f$

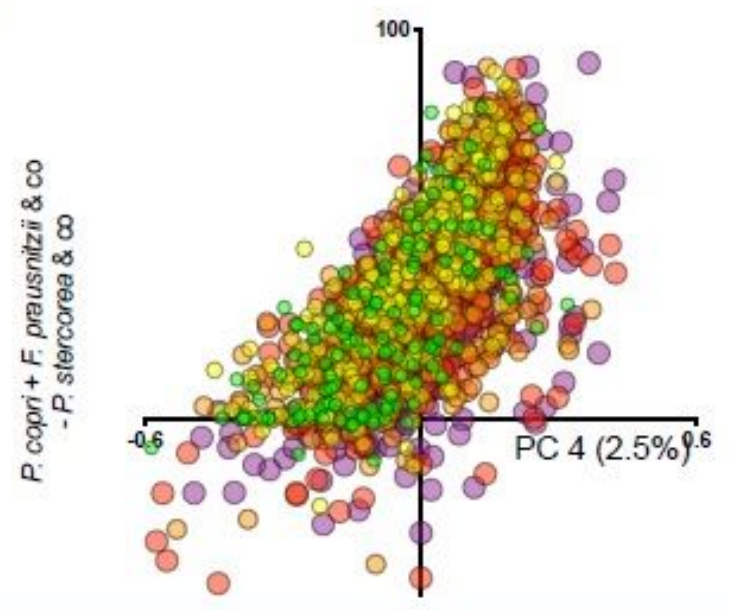




\section{Figure 6}

Maturation process analysed by Principal Component Analysis combined with Spearman rho correlation A Principal Component Analysis (PCA) combined with Spearman rho correlation analysis was performed to analyse the maturation process in more detail for different dominant taxa and bacterial networks. The correlations between F. prausnitzii and P. copri are shown in Figure 6.a. Figure 6.b shows the correlation between P. copri and P. stercorea \& co cluster. Principal component 1 (PC1) in Figure 6.c represents $73.5 \%$ of the variation in the data and indicates the maturation process to be the accumulation of Prevotella copri and both the F. prausnitzii and P. stercorea trophic networks over time. The build-up of the trophic networks over time is furthermore well described by PC3 in Figure 6.d. PC2 in Figure 6.e indicates how P. copri and the P. stercorea network are negatively correlated with Bacteroides whilst the F. prausnitzii network is positively correlated with Bacteroides. PC4 in Figure 6.f indicates that P.copri and the F. prausnitzii network are positively correlated with one another

\section{Supplementary Files}

This is a list of supplementary files associated with this preprint. Click to download.

- TheGamibaGutMicrobiomeOnlineSupplement.docx

- SupplementaryTable1.jpg

- SupplementaryTable2RankTestforthe11agegroups.pdf

- SupplementaryTable3.jpg

- SupplementaryFigure1.jpg

- SupplementaryFigure2.jpg

- SupplementaryFigure3Pairedtimepointsanalysis.pdf

- SupplementaryFigure4.jpg

- SupplementaryFigure5MLRAlphaindexwithageWAZHAZWHZ.pdf 